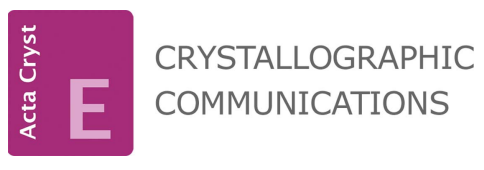

ISSN 2056-9890

Received 1 February 2018

Accepted 19 February 2018

Edited by J. T. Mague, Tulane University, USA

Keywords: benzoxathiol-2-one; hydrogen bonds; Hirshfeld surface; crystal structure.

CCDC references: $1486983 ; 1486982$; 1486987

Supporting information: this article has supporting information at journals.iucr.org/e

\section{Different molecular conformations in the crystal structures of three 5-nitroimidazolyl derivatives}

\author{
Luis F. B. Osorio, ${ }^{a}$ Samir A. Carvalho, ${ }^{a}$ Edson F. da Silva, ${ }^{a}$ Carlos A. M. Fraga, ${ }^{b}$ \\ Solange M. S. V. Wardell, ${ }^{c}$ Bruce F. Milne, ${ }^{d}$ James L. Wardell ${ }^{\mathrm{e}}$ and William T. A. \\ Harrison $^{\mathbf{e} *}$
}

\begin{abstract}
anstituto de Tecnologia em Fármacos e Farmanguinhos, Fundação Oswaldo Cruz, 21041-250 Rio de Janeiro, RJ, Brazil, ${ }^{\mathbf{b}}$ Programa de Pesquisa em Desenvolvimento de Fármacos, Instituto de Ciências Biomédicas, Universidade Federal do Rio de Janeiro, PO Box 68023, 21941-902 Rio de Janeiro, RJ, Brazil, ' $\mathrm{CHEMSOL}, 1$ Harcourt Road, Aberdeen AB15 5NY, Scotland, ${ }^{\mathbf{d}}$ CFisUC, Physics Department, University of Coimbra, Rua Larga 3004-516, Coimbra, Portugal, and ${ }^{\mathbf{e}}$ Department of Chemistry, University of Aberdeen, Meston Walk, Aberdeen AB24 3UE, Scotland. *Correspondence e-mail: w.harrison@abdn.ac.uk
\end{abstract}

The crystal structures of $(E)$-1-methyl-5-nitro-1 $H$-imidazole-2-carbaldehyde $O$-benzyloxime, $\mathrm{C}_{12} \mathrm{H}_{12} \mathrm{~N}_{4} \mathrm{O}_{3}$, (I), (E)-1-methyl-5-nitro- $1 H$-imidazole-2-carbaldehyde $O$-(4-fluorobenzyl) oxime, $\mathrm{C}_{12} \mathrm{H}_{11} \mathrm{FN}_{4} \mathrm{O}_{3}$, (II), and (E)-1-methyl-5nitro- $1 H$-imidazole-2-carbaldehyde $O$-(4-bromobenzyl) oxime, $\mathrm{C}_{12} \mathrm{H}_{11} \mathrm{BrN}_{4} \mathrm{O}_{3}$, (III), are described. The dihedral angle between the ring systems in (I) is $49.66(5)^{\circ}$ and the linking $\mathrm{N}_{\mathrm{m}}-\mathrm{C}-\mathrm{C}=\mathrm{N}(\mathrm{m}=$ methylated) bond shows an anti conformation [torsion angle $=175.00(15)^{\circ}$ ]. Compounds (II) and (III) are isostructural [dihedral angle between the aromatic rings $=8.31(5)^{\circ}$ in (II) and $5.34(15)^{\circ}$ in (III)] and differ from (I) in showing a near-syn conformation for the $\mathrm{N}_{\mathrm{m}}-\mathrm{C}-\mathrm{C}=\mathrm{N}$ linker [torsion angles for (II) and (III) $=17.64$ (18) and $8.7(5)^{\circ}$, respectively], which allows for the occurrence of a short intramolecular $\mathrm{C}-$ $\mathrm{H} \cdots \mathrm{N}$ contact. In the crystal of $(\mathrm{I}), \mathrm{C}-\mathrm{H} \cdots \mathrm{N}$ hydrogen bonds link the molecules into [010] chains, which are cross-linked by very weak $\mathrm{C}-\mathrm{H} \cdots \mathrm{O}$ bonds into (100) sheets. Weak aromatic $\pi-\pi$ stacking interactions occur between the sheets. The extended structures of (II) and (III) feature several C $-\mathrm{H} \cdots \mathrm{N}$ and $\mathrm{C}-\mathrm{H} \cdots \mathrm{O}$ hydrogen bonds, which link the molecules into threedimensional networks, which are consolidated by aromatic $\pi-\pi$ stacking interactions. Conformational energy calculations and Hirshfeld fingerprint analyses for (I), (II) and (III) are presented and discussed.

\section{Chemical context}

Trypanosomes infect a variety of hosts and cause various serious illnesses, including sleeping sickness (transmitted by Trypanosoma brucei) and Chagas' disease. The infectious agent of Chagas' disease is the protozoan parasite Trypanosoma cruzi, which produces progressive symptoms from mild swelling to intestinal disease and ultimately heart failure (Rassi et al., 2010). New effective drugs are urgently required for the treatment of Chagas' disease, which infects an estimated 6.6 million people worldwide (Rassi et al., 2010): benznidazole and nifurtimox have been the only recognised treatments for over 40 years and both drugs present variable results and undesirable side effects (Soeiro \& Castro, 2011). Megazol, while active, also has serious side effects (Poli et al. 2002).

We have recently described (Carvalho et al., 2017) the syntheses and biological activities of a family of 5-nitroimidazolyl- $O$-benzyloxime ethers, which displayed moderate 
antitrypanosidal activity. We now report the crystal structures, Hirshfeld surface analyses and conformational energy calculations for three compounds from that study, viz. (E)-1methyl-5-nitro- $1 H$-imidazole-2-carbaldehyde $O$-benzyloxime, $\mathrm{C}_{12} \mathrm{H}_{12} \mathrm{~N}_{4} \mathrm{O}_{3}$ (I), (E)-1-methyl-5-nitro-1H-imidazole-2-carbaldehyde $O$-(4-fluorobenzyl) oxime $\mathrm{C}_{12} \mathrm{H}_{11} \mathrm{FN}_{4} \mathrm{O}_{3}$ (II) and (E)1-methyl-5-nitro- $1 \mathrm{H}$-imidazole-2-carbaldehyde $\mathrm{O}$-(4-bromobenzyl) oxime, $\mathrm{C}_{12} \mathrm{H}_{11} \mathrm{BrN}_{4} \mathrm{O}_{3}$ (III).

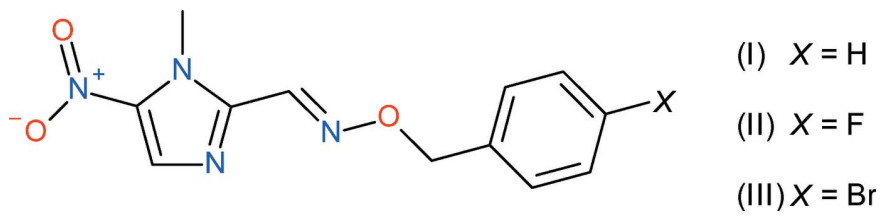

\section{Structural commentary}

Compound (I) crystallizes in space group $P 2_{1} / c$ with one molecule in the asymmetric unit (Fig. 1 and Table 1). The dihedral angle between the imidazole ring $(\mathrm{C} 1 / \mathrm{C} 2 / \mathrm{C} 3 / \mathrm{N} 1 / \mathrm{N} 2)$ and phenyl group (C7-C12) is $49.66(5)^{\circ}$. The $\mathrm{N} 4 / \mathrm{O} 2 / \mathrm{O} 3$ nitro group is approximately coplanar with its attached ring [dihedral angle $\left.=7.87(17)^{\circ}\right]$. The $\mathrm{C}-\mathrm{C}$ and $\mathrm{C}-\mathrm{N}$ bond lengths within the heterocyclic ring show typical values and $\mathrm{N} 2$ is statistically planar $\left(\right.$ bond-angle sum $=359.7^{\circ}$ ). The angle $\mathrm{C} 1-$ $\mathrm{N} 2-\mathrm{C} 4\left[129.47(13)^{\circ}\right]$ is significantly greater than $\mathrm{C} 3-\mathrm{N} 2-$ $\mathrm{C} 4\left[126.14(14)^{\circ}\right]$ perhaps because of steric repulsion between the $\mathrm{C} 4$ methyl group and the nitro group. The key parameter defining the conformation of the molecule of (I) is the N2$\mathrm{C} 3-\mathrm{C} 5=\mathrm{N} 3$ torsion angle: the value of $175.00(15)^{\circ}$ indicates an anti conformation for these atoms. The rest of the chain linking the rings can be described as extended in terms of the $\mathrm{C} 3-\mathrm{C} 5=\mathrm{N} 3-\mathrm{O} 1, \mathrm{C} 5=\mathrm{N} 3-\mathrm{O} 1-\mathrm{C} 6$ and $\mathrm{N} 3-\mathrm{O} 1-\mathrm{C} 6-\mathrm{C} 7$ torsion angles of $175.55(14),-172.50(15)$ and $172.62(14)^{\circ}$, respectively. The major twist in the molecule of (I) occurs about the $\mathrm{C} 6-\mathrm{C} 7$ bond as indicated by the $\mathrm{O} 1-\mathrm{C} 6-\mathrm{C} 7-$ $\mathrm{C} 12$ torsion angle of $-45.5(2)^{\circ}$. Assuming that the rotatinggroup refinement model for the $\mathrm{C} 4$ methyl group is reliable, it may be seen that this group has twisted about the $\mathrm{N} 2-\mathrm{C} 4$ bond to reduce steric repulsion with $\mathrm{H} 5$, although a rather short intramolecular contact $(\mathrm{H} 5 \cdots \mathrm{H} 4 \mathrm{C}=2.12 \AA)$ is still present.

Compounds (II) and (III) are isostructural, crystallizing in $P 2_{1} / n$ with one molecule in the asymmetric unit (Figs. 2 and 3 ). The dihedral angles between the aromatic rings for (II) and (III) are $8.31(5)$ and $5.34(15)^{\circ}$, respectively, whereas the

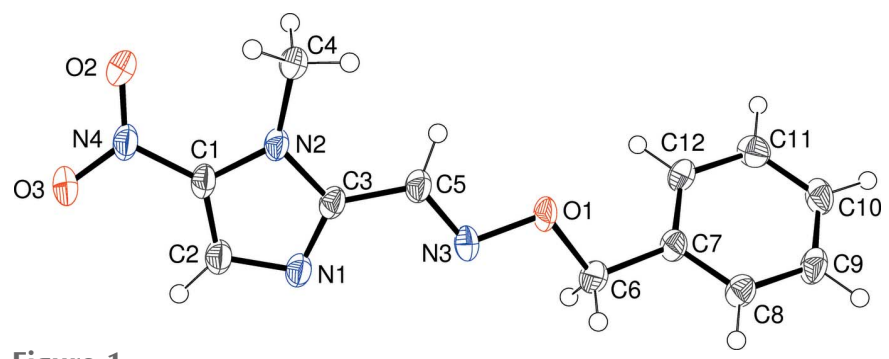

Figure 1

The molecular structure of (I) showing 50\% displacement ellipsoids.

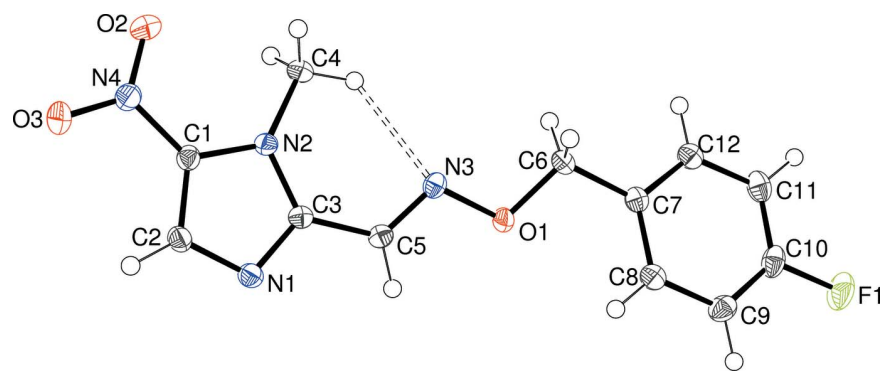

Figure 2

The molecular structure of (II) showing 50\% displacement ellipsoids.

dihedral angles for the nitro group and its attached ring are $2.83(11)$ and $5.9(30)^{\circ}$, respectively. The geometrical data for the imidazole rings in (II) and (III) show no significant differences compared to (I) but a major conformational difference is seen in terms of the $\mathrm{N} 2-\mathrm{C} 3-\mathrm{C} 5=\mathrm{N} 3$ torsion angles of 17.64 (18) for (II) and 8.7 (5) for (III), indicating an approximate syn conformation, as opposed to anti for (I). This reorientation facilitates the formation of an intramolecular $\mathrm{C} 4-\mathrm{H} 4 \mathrm{C} \cdots \mathrm{N} 3$ hydrogen bond in both (II) (Table 2) and (III) (Table 3). The rest of the linking chain displays an extended conformation in both (II) and (III) with respective $\mathrm{C} 3-$ $\mathrm{C} 5=\mathrm{N} 3-\mathrm{O} 1, \quad \mathrm{C} 5=\mathrm{N} 3-\mathrm{O} 1-\mathrm{C} 6 \quad$ and $\mathrm{N} 3-\mathrm{O} 1-\mathrm{C} 6-\mathrm{C} 7$ torsion angles of 179.79 (9), -173.96 (9) and 175.61 (8) in (II) and $179.2(2),-171.8(2)$ and 179.7 (2) in (III). The C6-C7 bond in (II) and (III) is somewhat less twisted than in (I), with $\mathrm{O} 1-\mathrm{C} 6-\mathrm{C} 7-\mathrm{C} 8$ torsion angles of $-30.95(14)$ and $-23.1(4)^{\circ}$ for (II) and (III), respectively.

\section{Computational calculations}

The different conformations of (I) compared to (II) and (III) were investigated by computational means. All calculations were performed with the Orca software package version 4.0.0.2 (Neese, 2012). Geometry optimizations were performed at the spin-component-scaled MP2 (SCS-MP2) level (Grimme, 2003) using the Def2-TZVP (Hellweg et al., 2007) basis set. Optimized geometries were then subjected to single-point energy calculations at the SCS-MP2 level with the larger Def2-QZVPP basis set to obtain final relative conformational energies. Geometry optimizations and single point energies were repeated using the SMD method to model the methanol solvent environment (Marenich et al., 2009) used in the crystallization experiments. The results (Table 4) show that

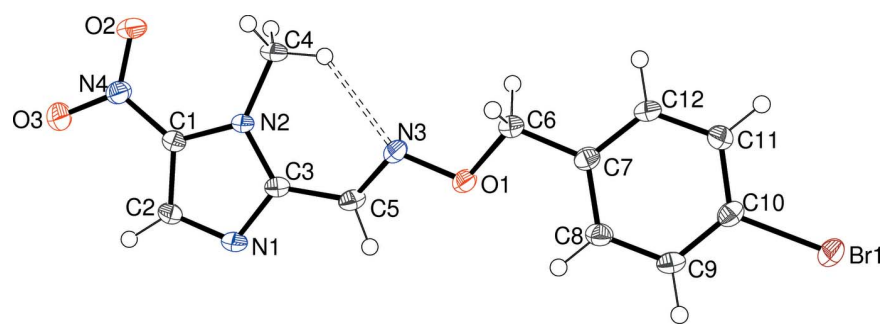

Figure 3

The molecular structure of (III) showing 50\% displacement ellipsoids. 
Table 1

Hydrogen-bond geometry $\left(\AA{ }^{\circ}\right)$ for $(\mathrm{I})$.

\begin{tabular}{lllll}
\hline$D-\mathrm{H} \cdots A$ & $D-\mathrm{H}$ & $\mathrm{H} \cdots A$ & $D \cdots A$ & $D-\mathrm{H} \cdots A$ \\
\hline $\mathrm{C} 4-\mathrm{H} 4 B \cdots \mathrm{N} 3^{\mathrm{i}}$ & 0.98 & 2.51 & $3.466(2)$ & 165 \\
$\mathrm{C} 5-\mathrm{H} 5 \cdots 3^{\mathrm{ii}}$ & 0.95 & 2.65 & $3.175(2)$ & 115 \\
\hline
\end{tabular}

Symmetry codes: (i) $-x, y+\frac{1}{2},-z+\frac{1}{2}$; (ii) $x,-y+\frac{1}{2}, z+\frac{1}{2}$.

Table 2

Hydrogen-bond geometry $\left(\AA,^{\circ}\right)$ for (II).

\begin{tabular}{lllll}
\hline$D-\mathrm{H} \cdots A$ & $D-\mathrm{H}$ & $\mathrm{H} \cdots A$ & $D \cdots A$ & $D-\mathrm{H} \cdots A$ \\
\hline $\mathrm{C} 4-\mathrm{H} 4 C \cdots \mathrm{N} 3$ & 0.98 & 2.29 & $3.0184(15)$ & 131 \\
$\mathrm{C} 4-\mathrm{H} 4 A \cdots \mathrm{N} 1^{\mathrm{i}}$ & 0.98 & 2.63 & $3.5693(16)$ & 160 \\
$\mathrm{C} 9-\mathrm{H} 9 \cdots \mathrm{N} 1^{\text {ii }}$ & 0.95 & 2.58 & $3.4973(16)$ & 163 \\
$\mathrm{C} 2-\mathrm{H} 2 \cdots \mathrm{O} 3^{\text {iii }}$ & 0.95 & 2.49 & $3.3165(15)$ & 145 \\
$\mathrm{C} 5-\mathrm{H} 5 \cdots \mathrm{O} 2^{\text {iv }}$ & 0.95 & 2.63 & $3.1676(14)$ & 116 \\
$\mathrm{C} 6-\mathrm{H} 6 A \cdots \mathrm{O} 2^{\mathrm{v}}$ & 0.99 & 2.54 & $3.1376(14)$ & 119 \\
$\mathrm{C} 4-\mathrm{H} 4 C \cdots \mathrm{F} 1^{\text {vi }}$ & 0.98 & 2.77 & $3.353(2)$ & 119 \\
\hline
\end{tabular}

Symmetry codes: (i) $\quad-x+\frac{1}{2}, y+\frac{1}{2},-z+\frac{1}{2}$; $\quad$ (ii) $\quad x-\frac{1}{2},-y+\frac{1}{2}, z+\frac{1}{2}$; $\quad$ (iii) $-x+1,-y+1,-z ; \quad$ (iv) $\quad-x+\frac{1}{2}, y-\frac{1}{2},-z+\frac{1}{2}$; $\quad$ (v) $\quad x-\frac{1}{2},-y+\frac{3}{2}, z+\frac{1}{2}$; $\quad$ (vi) $-x+\frac{1}{2}, y+\frac{1}{2},-z+\frac{3}{2}$.

Table 3

Hydrogen-bond geometry $\left(\AA,^{\circ}\right)$ for (III).

\begin{tabular}{lllll}
\hline$D-\mathrm{H} \cdots A$ & $D-\mathrm{H}$ & $\mathrm{H} \cdots A$ & $D \cdots A$ & $D-\mathrm{H} \cdots A$ \\
\hline $\mathrm{C} 4-\mathrm{H} 4 C \cdots \mathrm{N} 3$ & 0.98 & 2.24 & $2.997(4)$ & 133 \\
$\mathrm{C} 4-\mathrm{H} 4 A \cdots \mathrm{N} 1^{\mathrm{i}}$ & 0.98 & 2.62 & $3.499(4)$ & 149 \\
$\mathrm{C} 9-\mathrm{H} 9 \cdots \mathrm{N} 1^{\mathrm{ii}}$ & 0.95 & 2.77 & $3.681(4)$ & 160 \\
$\mathrm{C} 2-\mathrm{H} 2 \cdots \mathrm{O} 3^{\mathrm{iii}}$ & 0.95 & 2.44 & $3.282(4)$ & 148 \\
$\mathrm{C} 5-\mathrm{H} 5 \cdots \mathrm{O} 2^{\text {iv }}$ & 0.95 & 2.64 & $3.341(4)$ & 131 \\
$\mathrm{C} 6-\mathrm{H} 6 A \cdots \mathrm{O} 2^{\mathrm{v}}$ & 0.99 & 2.63 & $3.254(4)$ & 121 \\
$\mathrm{C} 4-\mathrm{H} 4 C \cdots \mathrm{Br} 1^{\mathrm{vi}}$ & 0.98 & 2.85 & $3.491(3)$ & 124
\end{tabular}

Symmetry codes: (i) $\quad-x+\frac{1}{2}, y+\frac{1}{2},-z+\frac{1}{2}$; (ii) $\quad x-\frac{1}{2},-y+\frac{1}{2}, z+\frac{1}{2}$; $\quad$ (iii) $-x+1,-y+1,-z$; (iv) $\quad-x+\frac{1}{2}, y-\frac{1}{2},-z+\frac{1}{2}$; $\quad$ (v) $\quad x-\frac{1}{2},-y+\frac{3}{2}, z+\frac{1}{2}$; $\quad$ (vi) $-x+\frac{1}{2}, y+\frac{1}{2},-z+\frac{3}{2}$

the syn conformation [i.e. that found for (II) and (III)] is favoured for all substituents by roughly the same energy (with the energy of the syn conformer arbitrarily defined to be zero in each case) either in vacuo or in a methanol solvent environment, although the differences in the latter case are quite small.

\section{Supramolecular features}

In the crystal of (I), the molecules are linked by $\mathrm{C}-\mathrm{H} \cdots \mathrm{N}$ hydrogen bonds (Table 1) to generate [010] C(6) chains, with adjacent molecules related by the $2_{1}$ screw axis (Fig. 4 ). The $\mathrm{C} 5-\mathrm{H} 5 \cdots \mathrm{O} 3$ contact is long and the angle is small, but if it is regarded as significant, it serves to cross-link the chains into (100) sheets. Weak aromatic $\pi-\pi$ stacking interactions arise between the sheets, such that each imidazole ring is sandwiched by two phenyl groups and vice versa [centroidcentroid separations $=3.7355$ (10) and 4.1184 (10) $\AA$; corresponding slippages $=1.35$ and $2.25 \AA$, respectively].

There are a number of intermolecular interactions in (II) (Table 2) and (III) (Table 3) and together they lead to threedimensional networks in each case. It is interesting that the $\mathrm{C} 9-\mathrm{H} 9 \cdots \mathrm{N} 1$ interaction in (II) is clearly a directional bond
Table 4

Relative conformational energies $\left(\mathrm{kJ} \mathrm{mol}^{-1}\right)$.

The two values refer to a vacuum and methanol solvation, respectively. The energy of the syn conformer is arbitrarily set to zero in each case.

\begin{tabular}{llll}
\hline Substituent & Compound & anti & syn \\
\hline $\mathrm{H}$ & (I) & $14.90 / 5.91$ & 0 \\
$\mathrm{CH}_{3}$ & Carvalho et al. (2017) & $14.90 / 6.84$ & 0 \\
$\mathrm{~F}$ & (II) & $17.12 / 6.17$ & 0 \\
$\mathrm{Br}$ & (III) & $16.84 / 6.17$ & 0 \\
\hline
\end{tabular}

$[\mathrm{H} \cdots \mathrm{N}=2.58 \AA$ compared to a van der Waals contact distance (Bondi, 1964) of $2.75 \AA$ for these atoms] whereas the equivalent contact in (III), included in Table 3 for completeness, has an $\mathrm{H} \cdots \mathrm{N}$ separation of $2.77 \AA$ and, by itself, would be very doubtful as a bond, which shows that isostructural crystals can show distinct variations in their weak interactions. This is supported by the presence of a weak $\mathrm{C} 4-\mathrm{H} 4 \mathrm{C} \cdots \mathrm{Br} 1$ bond in (III) $(\mathrm{H} \cdots \mathrm{Br}=2.85 \AA$, van der Waals contact distance $=3.05 \AA$ ) whilst the equivalent link in (II) has $\mathrm{H} \cdot \mathrm{F}=2.77 \AA$, significantly greater than the van der Waals contact distance of $2.67 \AA$ and would not be regarded as a significant bond. As in (I), $\pi-\pi$ stacking appears to consolidate the crystals of (II) and (III), in which the imidazole rings and phenyl rings form alternating stacks, which propagate in [100]. In (II), the imidazole ring faces phenyl rings with centroid-centroid (slippage) distances of 3.7297 (7) (1.23) and 3.9323 (7) $\AA$ $(1.64 \AA$ ). Equivalent data for (III) are 3.7664 (18) (1.47) and

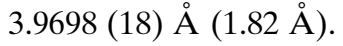

\section{Hirshfeld surface analysis}

Hirshfeld surface fingerprint plots for (I), (II) and (III) (supplementary Figs. 1, 2 and 3, respectively) were calculated with CrystalExplorer17 (Turner et al., 2017). When the fingerprint plots are decomposed into the separate types of intermolecular contacts (McKinnon et al., 2007), it may be

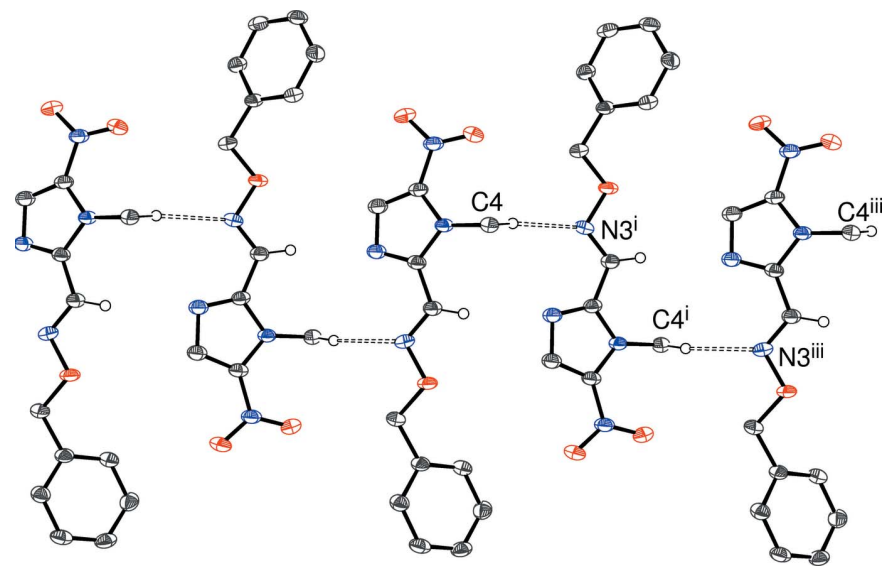

Figure 4

Fragment of an [010] hydrogen-bonded chain in the crystal of (I). Symmetry codes: (i) $-\mathrm{x}, \frac{1}{2}+y, 1 / 2-\mathrm{z}$; (iii) $x, y+1, z$. 
Table 5

Hirshfeld contact interactions (\%).

\begin{tabular}{llll}
\hline Contact type & $(\mathrm{I})$ & $(\mathrm{II})$ & $(\mathrm{III})$ \\
\hline $\mathrm{H} \cdots \mathrm{H}$ & 34.6 & 30.3 & 28.3 \\
$\mathrm{O} \cdots \mathrm{H} / \mathrm{H} \cdots \mathrm{O}$ & 24.6 & 24.4 & 23.2 \\
$\mathrm{~N} \cdots \mathrm{H} / \mathrm{H} \cdots \mathrm{N}$ & 14.7 & 9.4 & 8.1 \\
$\mathrm{C} \cdots \mathrm{H} / \mathrm{H} \cdots \mathrm{C}$ & 12.4 & 6.0 & 6.5 \\
$\mathrm{C} \cdots \mathrm{C}$ & 4.6 & 5.8 & 5.9 \\
$X \cdots \mathrm{H} / \mathrm{H} \cdots X$ & - & 11.7 & 15.0 \\
\hline
\end{tabular}

seen (Table 5) that as a percentage of surface interactions, $\mathrm{H} \cdot \mathrm{H}$ contacts (i.e. van der Waals interactions) are the most significant in each structure, followed by $\mathrm{O} \cdots \mathrm{H} / \mathrm{H} \cdots \mathrm{O}$ contacts. It is interesting the percentage of the latter for (I) is slightly higher than for (II), despite the fact that (I) features one weak $\mathrm{C}-\mathrm{H} \cdots \mathrm{O}$ bond at best whilst (II) features three such bonds. The C . C contacts (associated with aromatic $\pi-\pi$ stacking) contribute a very small percentage in each structure, which is slightly surprising given the significant $\pi-\pi$ stacking interactions noted above. Finally, it may be noted that the $\mathrm{C} \cdots \mathrm{H} / \mathrm{H} \cdots \mathrm{C}$ and $\mathrm{N} \cdots \mathrm{N} / \mathrm{H} \cdots \mathrm{N}$ contributions for (I) and the $\mathrm{C} \cdots \mathrm{H} / \mathrm{H} \cdots \mathrm{C}, \mathrm{N} \cdots \mathrm{N} / \mathrm{H} \cdots \mathrm{N}$ and $X-\mathrm{H} / \mathrm{H} \cdots X$ contributions for (II) and (III) sum to approximately the same amount.

Beyond a vague appeal to 'packing forces', we find it difficult to explain why (I) forms the energetically disfavoured anti conformation in the crystal: it allows the $\mathrm{C} 5-\mathrm{H} 5$ group to form a weak hydrogen bond (Table 1) to a nitro group oxygen atom but it should be noted that the same grouping forms a similar bond in the opposite direction (i.e. pointing away from C4) in both (II) and (III). The syn conformation for (II) and (III) seems to be favoured in terms of the occurrence of an intramolecular $\mathrm{C}-\mathrm{H} \cdots \mathrm{N}$ link and it is possible that weak $\mathrm{C}-$ $\mathrm{H} \cdots X(X=\mathrm{F}, \mathrm{Br})$ interactions in the crystals of (II) and (III) provide some stabilization not possible in (I), although they are at the opposite end of the molecule. The Hirshfeld fingerprint data (Table 5) show that $\mathrm{N} \cdots \mathrm{H} / \mathrm{H} \cdots \mathrm{N}$ and $\mathrm{C} \cdots \mathrm{H} /$ $\mathrm{H}$. . C contacts are somewhat more significant in the crystal of (I) but the energetic consequences of these are not clear. We cannot rule out the posssibility that a polymorph of (I) may exist in which the $\mathrm{N}_{\mathrm{m}}-\mathrm{C}-\mathrm{C}=\mathrm{N}$ grouping has a syn conformation but with a different overall packing motif to (II) and (III).

\section{Database survey}

A survey of of the Cambridge Structural Database (Groom et al., 2016: updated to January 2018) for the 1-methyl 5-nitro imidazole fragment revealed 33 hits. The 4-methyl-substituted analogue of the title compounds, $N$-[(4-methylbenzyl)oxy]-1(1-methyl-5-nitro-1H-imidazol-2-yl)methanimine (refcode: TEVGAF), has been reported by Carvalho et al. (2017): its $\mathrm{N}_{\mathrm{m}}-\mathrm{C}-\mathrm{C}=\mathrm{N}$ torsion angle is $-30.7(2)^{\circ}$, i.e. somewhat twisted from syn.

Table 6

Experimental details.

(I)

Crystal data

Chemical formula

$M_{\mathrm{r}}$

Crystal system, space group

Temperature (K)

$a, b, c(\AA)$

$\beta\left({ }^{\circ}\right)$

$V\left(\AA^{3}\right)$

$Z$

Radiation type

$\mu\left(\mathrm{mm}^{-1}\right)$

Crystal size (mm)

Data collection

Diffractometer

Absorption correction

$T_{\min }, T_{\max }$

No. of measured, independent and observed $[I>2 \sigma(I)]$ reflections

$R_{\text {int }}$

$(\sin \theta / \lambda)_{\max }\left(\AA^{-1}\right)$

0.055
0.649

Refinement

$R\left[F^{2}>2 \sigma\left(F^{2}\right)\right], w R\left(F^{2}\right), S$

No. of reflections

No. of parameters

$\mathrm{H}$-atom treatment

$\Delta \rho_{\max }, \Delta \rho_{\min }\left(\mathrm{e} \AA^{-3}\right)$

(II)

(III)

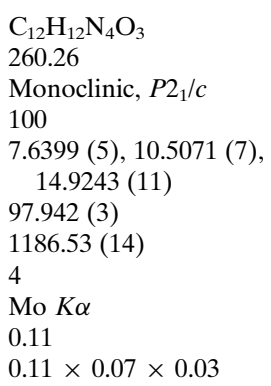

$\mathrm{C}_{12} \mathrm{H}_{11} \mathrm{FN}_{4} \mathrm{O}_{3}$
278.25
Monoclinic, $P 2_{1} / n$
120
$7.5484(2), 12.6442(4), 13.4150(9)$
$102.988(7)$
$1247.62(10)$
4
Mo $K \alpha$
0.12
$0.19 \times 0.13 \times 0.10$

$\mathrm{C}_{12} \mathrm{H}_{11} \mathrm{BrN}_{4} \mathrm{O}_{3}$

339.16

Monoclinic, $P 2_{1} / n$

120

7.6024 (2), 12.7526 (3), 13.8954 (5)

$104.869(2)$

$1302.05(7)$

4

Mo $K \alpha$

3.17

$0.66 \times 0.52 \times 0.24$

Rigaku Saturn724+ CCD

Multi-scan (FS_ABSCOR; Rigaku, 2013)

$0.618,1.000$

$7757,2705,1891$

0.055
0.649

$0.047,0.126,0.95$

2705

173

$\mathrm{H}$-atom parameters constrained

$0.24,-0.22$
Rigaku Saturn724+ CCD

Multi-scan (FS_ABSCOR; Rigaku, 2013)

$0.802,1.000$

$8522,2848,2292$

0.021

0.649

$0.034,0.094,1.09$

2848

182

H-atom parameters constrained

$0.29,-0.18$
Rigaku Mercury CCD

Multi-scan (FS_ABSCOR; Rigaku, 2013)

$0.438,1.000$

16791, 2974, 2835

0.065

0.650

$0.051,0.140,1.11$

2974

182

$\mathrm{H}$-atom parameters constrained $1.78,-1.03$ 2010). 


\section{Synthesis and crystallization}

The syntheses and spectroscopic data of the title compounds have already been described (Carvalho et al., 2017). The crystals used for data collections in this study were recrystallized from methanol solution in each case as colourless plates of (I), orange blocks of (II) and yellow blocks of (III).

\section{Refinement}

Crystal data, data collection and structure refinement details are summarized in Table 6. The hydrogen atoms were geometrically placed $(\mathrm{C}-\mathrm{H}=0.95-0.99 \AA)$ and refined as riding atoms. The constraint $U_{\text {iso }}(\mathrm{H})=1.2 U_{\text {eq }}$ (carrier) or $1.5 U_{\text {eq }}$ (methyl carrier) was applied in all cases. The methyl groups were allowed to rotate, but not to tip, to best fit the electron density.

\section{Acknowledgements}

We thank the EPSRC National Crystallography Service (University of Southampton) for the X-ray data collections.

\section{References}

Bondi, A. (1964). J. Phys. Chem. 68, 441-451.
Carvalho, S. A., Osorio, L. F. B., Salomão, K., de Castro, S. L., Wardell, S. M. S. V., Wardell, J. L., da Silva, E. F. \& Fraga, C. A. M. (2017). J. Heterocycl. Chem. 54, 3626-3631.

Farrugia, L. J. (2012). J. Appl. Cryst. 45, 849-854.

Grimme, S. (2003). J. Chem. Phys. 118, 9095-9102.

Groom, C. R., Bruno, I. J., Lightfoot, M. P. \& Ward, S. C. (2016). Acta Cryst. B72, 171-179.

Hellweg, A., Hättig, C., Höfener, S. \& Klopper, W. (2007). Theor. Chem. Acc. 117, 587-597.

Marenich, A. V., Cramer, C. J. \& Truhlar, D. G. (2009). J. Phys. Chem. $B, \mathbf{1 1 3}, 6378-6396$.

McKinnon, J. J., Jayatilaka, D. \& Spackman, M. A. (2007). Chem. Commun. 3814-3816.

Neese, F. (2012). WIREs Comput. Mol. Sci. 2, 73-78.

Poli, P., Aline de Mello, M., Buschini, A., Mortara, R. A., Northfleet de Albuquerque, C., da Silva, S., Rossi, C. \& Zucchi, T. M. (2002). Biochem. Pharmacol. 64, 1617-1627.

Rassi, A. Jr, Rassi, A. \& Marin-Neto, J. A. (2010). Lancet, 375, 13881402.

Rigaku (2012). CrystalClear. Rigaku Corporation, Tokyo, Japan.

Rigaku (2013). FS_ABSCOR. Rigaku Corporation, Tokyo, Japan.

Sheldrick, G. M. (2008). Acta Cryst. A64, 112-122.

Sheldrick, G. M. (2015). Acta Cryst. C71, 3-8.

Soeiro, M. de N. C. \& de Castro, S. L. (2011). Open Med. Chem. J. 5, 21-30.

Turner, M. J., McKinnon, J. J., Wolff, S. K., Grimwood, D. J., Spackman, P. R., Jayatilaka, D. \& Spackman, M. A. (2017). CrystalExplorer17. University of Western Australia, Nedlands, Western Australia; http://hirshfeldsurface.net.

Westrip, S. P. (2010). J. Appl. Cryst. 43, 920-925. 


\section{supporting information}

Acta Cryst. (2018). E74, 380-384 [https://doi.org/10.1107/S2056989018002876]

\section{Different molecular conformations in the crystal structures of three 5-nitro- imidazolyl derivatives}

Luis F. B. Osorio, Samir A. Carvalho, Edson F. da Silva, Carlos A. M. Fraga, Solange M. S. V.

Wardell, Bruce F. Milne, James L. Wardell and William T. A. Harrison

Computing details

For all structures, data collection: CrystalClear (Rigaku, 2012); cell refinement: CrystalClear (Rigaku, 2012); data reduction: CrystalClear (Rigaku, 2012); program(s) used to solve structure: SHELXS97 (Sheldrick, 2008); program(s) used to refine structure: SHELXL2014 (Sheldrick, 2015); molecular graphics: ORTEP-3 for Windows (Farrugia, 2012); software used to prepare material for publication: publCIF (Westrip, 2010).

(E)-1-Methyl-5-nitro-1 H-imidazole-2-carbaldehyde O-benzyloxime (I)

Crystal data

$\mathrm{C}_{12} \mathrm{H}_{12} \mathrm{~N}_{4} \mathrm{O}_{3}$

$M_{r}=260.26$

Monoclinic, $P 2_{1} / c$

$a=7.6399(5) \AA$

$b=10.5071$ (7) $\AA$

$c=14.9243(11) \AA$

$\beta=97.942(3)^{\circ}$

$V=1186.53(14) \AA^{3}$

$Z=4$

$F(000)=544$

$D_{\mathrm{x}}=1.457 \mathrm{Mg} \mathrm{m}^{-3}$

Mo $K \alpha$ radiation, $\lambda=0.71073 \AA$

Cell parameters from 7971 reflections

$\theta=2.4-27.5^{\circ}$

$\mu=0.11 \mathrm{~mm}^{-1}$

$T=100 \mathrm{~K}$

Plate, colourless

$0.11 \times 0.07 \times 0.03 \mathrm{~mm}$

Data collection

Rigaku Saturn724+ CCD

2705 independent reflections

diffractometer

$\omega$ scans

1891 reflections with $I>2 \sigma(I)$

Absorption correction: multi-scan

$R_{\text {int }}=0.055$

(FS_ABSCOR; Rigaku, 2013)

$T_{\min }=0.618, T_{\max }=1.000$

$\theta_{\max }=27.5^{\circ}, \theta_{\min }=2.4^{\circ}$

$h=-9 \rightarrow 6$

$k=-13 \rightarrow 13$

7757 measured reflections

$l=-19 \rightarrow 18$

\section{Refinement}

Refinement on $F^{2}$

Least-squares matrix: full

$R\left[F^{2}>2 \sigma\left(F^{2}\right)\right]=0.047$

$w R\left(F^{2}\right)=0.126$

$S=0.95$

2705 reflections

173 parameters

0 restraints

Primary atom site location: structure-invariant direct methods

Hydrogen site location: inferred from neighbouring sites

$\mathrm{H}$-atom parameters constrained

$w=1 /\left[\sigma^{2}\left(F_{\mathrm{o}}^{2}\right)+(0.0674 P)^{2}\right]$

where $P=\left(F_{\mathrm{o}}^{2}+2 F_{\mathrm{c}}{ }^{2}\right) / 3$

$(\Delta / \sigma)_{\max }<0.001$ 
$\Delta \rho_{\max }=0.24$ e $\AA^{-3}$

$\Delta \rho_{\min }=-0.22$ e $\AA^{-3}$

Special details

Geometry. All esds (except the esd in the dihedral angle between two 1.s. planes) are estimated using the full covariance matrix. The cell esds are taken into account individually in the estimation of esds in distances, angles and torsion angles; correlations between esds in cell parameters are only used when they are defined by crystal symmetry. An approximate (isotropic) treatment of cell esds is used for estimating esds involving l.s. planes.

Fractional atomic coordinates and isotropic or equivalent isotropic displacement parameters $\left(\hat{A}^{2}\right)$

\begin{tabular}{|c|c|c|c|c|}
\hline & $x$ & $y$ & $z$ & $U_{\text {iso }} * / U_{\text {eq }}$ \\
\hline $\mathrm{C} 1$ & $-0.2026(2)$ & $0.18424(17)$ & $0.08287(11)$ & $0.0272(4)$ \\
\hline $\mathrm{C} 2$ & $-0.1242(2)$ & $0.06989(17)$ & $0.10516(11)$ & $0.0302(4)$ \\
\hline $\mathrm{H} 2$ & -0.1177 & 0.0010 & 0.0646 & $0.036^{*}$ \\
\hline $\mathrm{C} 3$ & $-0.0953(2)$ & $0.18453(17)$ & $0.22478(11)$ & $0.0262(4)$ \\
\hline $\mathrm{C} 4$ & $-0.2593(2)$ & $0.38590(16)$ & $0.17211(11)$ & 0.0295 \\
\hline $\mathrm{H} 4 \mathrm{~A}$ & -0.3780 & 0.3918 & 0.1378 & $0.044^{*}$ \\
\hline $\mathrm{H} 4 \mathrm{~B}$ & -0.1832 & 0.4512 & 0.1508 & $0.044^{*}$ \\
\hline $\mathrm{H} 4 \mathrm{C}$ & -0.2661 & 0.3991 & 0.2365 & $0.044^{*}$ \\
\hline $\mathrm{C} 5$ & $-0.0422(2)$ & $0.23260(16)$ & $0.31595(11)$ & 0.0277 (4) \\
\hline H5 & -0.0610 & 0.3193 & 0.3300 & $0.033^{*}$ \\
\hline C6 & $0.1846(3)$ & $0.13647(17)$ & $0.51983(12)$ & $0.0353(5)$ \\
\hline H6A & 0.2794 & 0.0969 & 0.4903 & $0.042^{*}$ \\
\hline H6B & 0.1069 & 0.0680 & 0.5372 & $0.042^{*}$ \\
\hline $\mathrm{C} 7$ & $0.2641(2)$ & $0.20824(16)$ & $0.60261(11)$ & $0.0275(4)$ \\
\hline $\mathrm{C} 8$ & $0.2637(2)$ & $0.15343(17)$ & $0.68727(11)$ & $0.0296(4)$ \\
\hline $\mathrm{H} 8$ & 0.2039 & 0.0751 & 0.6927 & $0.036^{*}$ \\
\hline C9 & $0.3508(2)$ & $0.21295(18)$ & $0.76444(12)$ & 0.0328 \\
\hline H9 & 0.3522 & 0.1739 & 0.8220 & $0.039^{*}$ \\
\hline $\mathrm{C} 10$ & $0.4346(2)$ & $0.32796(18)$ & $0.75770(12)$ & $0.0336(4)$ \\
\hline H10 & 0.4943 & 0.3680 & 0.8103 & $0.040^{*}$ \\
\hline C11 & $0.4313(2)$ & $0.38490(17)$ & $0.67342(12)$ & $0.0327(4)$ \\
\hline H11 & 0.4865 & 0.4652 & 0.6686 & $0.039^{*}$ \\
\hline $\mathrm{C} 12$ & $0.3475(2)$ & $0.32503(17)$ & $0.59602(11)$ & 0.0293 \\
\hline H12 & 0.3471 & 0.3639 & 0.5385 & $0.035^{*}$ \\
\hline N1 & $-0.05705(18)$ & $0.06958(14)$ & $0.19410(9)$ & 0.0292 \\
\hline N2 & $-0.18520(17)$ & $0.25946(13)$ & $0.15896(9)$ & $0.0257(3)$ \\
\hline N3 & $0.03028(18)$ & $0.15619(14)$ & $0.37682(9)$ & $0.0287(3)$ \\
\hline N4 & $-0.28663(19)$ & $0.22404(14)$ & $-0.00435(9)$ & 0.0308 \\
\hline $\mathrm{O} 1$ & $0.08352(16)$ & $0.22291(11)$ & $0.45775(7)$ & $0.0304(3)$ \\
\hline $\mathrm{O} 2$ & $-0.33264(17)$ & $0.33549(12)$ & $-0.01644(8)$ & $0.0382(3)$ \\
\hline $\mathrm{O} 3$ & $-0.30669(18)$ & $0.14106(13)$ & $-0.06357(8)$ & $0.0402(4)$ \\
\hline
\end{tabular}

Atomic displacement parameters $\left(\AA^{2}\right)$

\begin{tabular}{lllllll}
\hline & $U^{11}$ & $U^{22}$ & $U^{33}$ & $U^{12}$ & $U^{13}$ & $U^{23}$ \\
\hline C1 & $0.0262(8)$ & $0.0346(9)$ & $0.0192(8)$ & $-0.0040(7)$ & $-0.0031(6)$ & $0.0003(7)$ \\
C2 & $0.0309(9)$ & $0.0354(9)$ & $0.0224(9)$ & $-0.0024(8)$ & $-0.0026(7)$ & $-0.0007(7)$ \\
C3 & $0.0231(8)$ & $0.0319(9)$ & $0.0215(8)$ & $-0.0024(7)$ & $-0.0038(6)$ & $0.0032(7)$
\end{tabular}




\begin{tabular}{lllllll} 
C4 & $0.0303(9)$ & $0.0299(8)$ & $0.0257(9)$ & $0.0015(7)$ & $-0.0055(7)$ & $0.0003(7)$ \\
C5 & $0.0276(9)$ & $0.0319(8)$ & $0.0215(8)$ & $-0.0012(7)$ & $-0.0033(7)$ & $0.0000(7)$ \\
C6 & $0.0412(10)$ & $0.0328(9)$ & $0.0270(9)$ & $0.0019(8)$ & $-0.0131(8)$ & $0.0024(8)$ \\
C7 & $0.0243(8)$ & $0.0320(9)$ & $0.0232(8)$ & $0.0031(7)$ & $-0.0070(6)$ & $0.0001(7)$ \\
C8 & $0.0266(8)$ & $0.0335(9)$ & $0.0272(9)$ & $0.0006(7)$ & $-0.0018(7)$ & $0.0034(7)$ \\
C9 & $0.0328(9)$ & $0.0427(10)$ & $0.0211(9)$ & $0.0104(8)$ & $-0.0030(7)$ & $0.0024(8)$ \\
C10 & $0.0305(9)$ & $0.0404(10)$ & $0.0263(9)$ & $0.0069(8)$ & $-0.0090(7)$ & $-0.0072(8)$ \\
C11 & $0.0271(9)$ & $0.0317(9)$ & $0.0366(10)$ & $-0.0005(7)$ & $-0.0049(7)$ & $-0.0031(8)$ \\
C12 & $0.0285(9)$ & $0.0344(9)$ & $0.0230(9)$ & $0.0024(8)$ & $-0.0031(7)$ & $0.0037(7)$ \\
N1 & $0.0306(7)$ & $0.0339(8)$ & $0.0212(7)$ & $-0.0019(6)$ & $-0.0033(6)$ & $0.0012(6)$ \\
N2 & $0.0247(7)$ & $0.0305(7)$ & $0.0197(7)$ & $-0.0012(6)$ & $-0.0049(6)$ & $0.0002(6)$ \\
N3 & $0.0282(7)$ & $0.0355(8)$ & $0.0203(7)$ & $-0.0027(6)$ & $-0.0040(6)$ & $-0.0042(6)$ \\
N4 & $0.0321(8)$ & $0.0374(8)$ & $0.0202(7)$ & $-0.0036(7)$ & $-0.0054(6)$ & $0.0002(7)$ \\
O1 & $0.0334(7)$ & $0.0358(6)$ & $0.0185(6)$ & $0.0028(5)$ & $-0.0087(5)$ & $-0.0025(5)$ \\
O2 & $0.0460(8)$ & $0.0378(7)$ & $0.0271(7)$ & $0.0049(6)$ & $-0.0085(6)$ & $0.0048(6)$ \\
O3 & $0.0500(8)$ & $0.0428(7)$ & $0.0236(7)$ & $-0.0058(6)$ & $-0.0097(6)$ & $-0.0054(6)$ \\
& & & & & & \\
\hline
\end{tabular}

Geometric parameters $\left(\AA,{ }^{\circ}\right)$

\begin{tabular}{|c|c|c|c|}
\hline $\mathrm{C} 1-\mathrm{C} 2$ & $1.363(2)$ & C6-H6A & 0.9900 \\
\hline $\mathrm{C} 1-\mathrm{N} 2$ & $1.375(2)$ & $\mathrm{C} 6-\mathrm{H} 6 \mathrm{~B}$ & 0.9900 \\
\hline $\mathrm{C} 1-\mathrm{N} 4$ & $1.432(2)$ & $\mathrm{C} 7-\mathrm{C} 8$ & $1.389(2)$ \\
\hline $\mathrm{C} 2-\mathrm{N} 1$ & $1.355(2)$ & $\mathrm{C} 7-\mathrm{C} 12$ & $1.392(2)$ \\
\hline $\mathrm{C} 2-\mathrm{H} 2$ & 0.9500 & $\mathrm{C} 8-\mathrm{C} 9$ & $1.396(2)$ \\
\hline $\mathrm{C} 3-\mathrm{N} 1$ & $1.338(2)$ & $\mathrm{C} 8-\mathrm{H} 8$ & 0.9500 \\
\hline $\mathrm{C} 3-\mathrm{N} 2$ & $1.368(2)$ & $\mathrm{C} 9-\mathrm{C} 10$ & $1.378(3)$ \\
\hline $\mathrm{C} 3-\mathrm{C} 5$ & $1.456(2)$ & $\mathrm{C} 9-\mathrm{H} 9$ & 0.9500 \\
\hline $\mathrm{C} 4-\mathrm{N} 2$ & $1.468(2)$ & $\mathrm{C} 10-\mathrm{C} 11$ & $1.390(3)$ \\
\hline $\mathrm{C} 4-\mathrm{H} 4 \mathrm{~A}$ & 0.9800 & $\mathrm{C} 10-\mathrm{H} 10$ & 0.9500 \\
\hline $\mathrm{C} 4-\mathrm{H} 4 \mathrm{~B}$ & 0.9800 & $\mathrm{C} 11-\mathrm{C} 12$ & $1.391(2)$ \\
\hline $\mathrm{C} 4-\mathrm{H} 4 \mathrm{C}$ & 0.9800 & $\mathrm{C} 11-\mathrm{H} 11$ & 0.9500 \\
\hline $\mathrm{C} 5-\mathrm{N} 3$ & $1.279(2)$ & $\mathrm{C} 12-\mathrm{H} 12$ & 0.9500 \\
\hline $\mathrm{C} 5-\mathrm{H} 5$ & 0.9500 & $\mathrm{~N} 3-\mathrm{O} 1$ & $1.4071(16)$ \\
\hline $\mathrm{C} 6-\mathrm{O} 1$ & $1.4428(19)$ & $\mathrm{N} 4-\mathrm{O} 2$ & $1.2287(18)$ \\
\hline $\mathrm{C} 6-\mathrm{C} 7$ & $1.502(2)$ & $\mathrm{N} 4-\mathrm{O} 3$ & $1.2359(18)$ \\
\hline $\mathrm{C} 2-\mathrm{C} 1-\mathrm{N} 2$ & $108.43(14)$ & $\mathrm{C} 12-\mathrm{C} 7-\mathrm{C} 6$ & $121.42(15)$ \\
\hline $\mathrm{C} 2-\mathrm{C} 1-\mathrm{N} 4$ & $127.30(16)$ & $\mathrm{C} 7-\mathrm{C} 8-\mathrm{C} 9$ & $120.27(17)$ \\
\hline $\mathrm{N} 2-\mathrm{C} 1-\mathrm{N} 4$ & $124.26(15)$ & $\mathrm{C} 7-\mathrm{C} 8-\mathrm{H} 8$ & 119.9 \\
\hline $\mathrm{N} 1-\mathrm{C} 2-\mathrm{C} 1$ & $109.59(15)$ & $\mathrm{C} 9-\mathrm{C} 8-\mathrm{H} 8$ & 119.9 \\
\hline $\mathrm{N} 1-\mathrm{C} 2-\mathrm{H} 2$ & 125.2 & $\mathrm{C} 10-\mathrm{C} 9-\mathrm{C} 8$ & $120.40(16)$ \\
\hline $\mathrm{C} 1-\mathrm{C} 2-\mathrm{H} 2$ & 125.2 & $\mathrm{C} 10-\mathrm{C} 9-\mathrm{H} 9$ & 119.8 \\
\hline $\mathrm{N} 1-\mathrm{C} 3-\mathrm{N} 2$ & $112.68(14)$ & $\mathrm{C} 8-\mathrm{C} 9-\mathrm{H} 9$ & 119.8 \\
\hline $\mathrm{N} 1-\mathrm{C} 3-\mathrm{C} 5$ & $125.95(14)$ & $\mathrm{C} 9-\mathrm{C} 10-\mathrm{C} 11$ & $119.54(16)$ \\
\hline $\mathrm{N} 2-\mathrm{C} 3-\mathrm{C} 5$ & $121.28(15)$ & $\mathrm{C} 9-\mathrm{C} 10-\mathrm{H} 10$ & 120.2 \\
\hline $\mathrm{N} 2-\mathrm{C} 4-\mathrm{H} 4 \mathrm{~A}$ & 109.5 & $\mathrm{C} 11-\mathrm{C} 10-\mathrm{H} 10$ & 120.2 \\
\hline $\mathrm{N} 2-\mathrm{C} 4-\mathrm{H} 4 \mathrm{~B}$ & 109.5 & $\mathrm{C} 10-\mathrm{C} 11-\mathrm{C} 12$ & $120.36(17)$ \\
\hline $\mathrm{H} 4 \mathrm{~A}-\mathrm{C} 4-\mathrm{H} 4 \mathrm{~B}$ & 109.5 & $\mathrm{C} 10-\mathrm{C} 11-\mathrm{H} 11$ & 119.8 \\
\hline
\end{tabular}




\begin{tabular}{|c|c|c|c|}
\hline $\mathrm{N} 2-\mathrm{C} 4-\mathrm{H} 4 \mathrm{C}$ & 109.5 & $\mathrm{C} 12-\mathrm{C} 11-\mathrm{H} 11$ & 119.8 \\
\hline $\mathrm{H} 4 \mathrm{~A}-\mathrm{C} 4-\mathrm{H} 4 \mathrm{C}$ & 109.5 & $\mathrm{C} 11-\mathrm{C} 12-\mathrm{C} 7$ & $120.16(16)$ \\
\hline $\mathrm{H} 4 \mathrm{~B}-\mathrm{C} 4-\mathrm{H} 4 \mathrm{C}$ & 109.5 & $\mathrm{C} 11-\mathrm{C} 12-\mathrm{H} 12$ & 119.9 \\
\hline $\mathrm{N} 3-\mathrm{C} 5-\mathrm{C} 3$ & $118.90(15)$ & $\mathrm{C} 7-\mathrm{C} 12-\mathrm{H} 12$ & 119.9 \\
\hline $\mathrm{N} 3-\mathrm{C} 5-\mathrm{H} 5$ & 120.5 & $\mathrm{C} 3-\mathrm{N} 1-\mathrm{C} 2$ & $105.18(14)$ \\
\hline $\mathrm{C} 3-\mathrm{C} 5-\mathrm{H} 5$ & 120.5 & $\mathrm{C} 3-\mathrm{N} 2-\mathrm{C} 1$ & $104.12(14)$ \\
\hline $\mathrm{O} 1-\mathrm{C} 6-\mathrm{C} 7$ & $109.39(14)$ & $\mathrm{C} 3-\mathrm{N} 2-\mathrm{C} 4$ & $126.14(14)$ \\
\hline $\mathrm{O} 1-\mathrm{C} 6-\mathrm{H} 6 \mathrm{~A}$ & 109.8 & $\mathrm{C} 1-\mathrm{N} 2-\mathrm{C} 4$ & $129.47(13)$ \\
\hline $\mathrm{C} 7-\mathrm{C} 6-\mathrm{H} 6 \mathrm{~A}$ & 109.8 & $\mathrm{C} 5-\mathrm{N} 3-\mathrm{O} 1$ & $110.01(13)$ \\
\hline $\mathrm{O} 1-\mathrm{C} 6-\mathrm{H} 6 \mathrm{~B}$ & 109.8 & $\mathrm{O} 2-\mathrm{N} 4-\mathrm{O} 3$ & $124.23(14)$ \\
\hline $\mathrm{C} 7-\mathrm{C} 6-\mathrm{H} 6 \mathrm{~B}$ & 109.8 & $\mathrm{O} 2-\mathrm{N} 4-\mathrm{C} 1$ & $119.68(14)$ \\
\hline $\mathrm{H} 6 \mathrm{~A}-\mathrm{C} 6-\mathrm{H} 6 \mathrm{~B}$ & 108.2 & $\mathrm{O} 3-\mathrm{N} 4-\mathrm{C} 1$ & $116.09(14)$ \\
\hline $\mathrm{C} 8-\mathrm{C} 7-\mathrm{C} 12$ & $119.23(15)$ & $\mathrm{N} 3-\mathrm{O} 1-\mathrm{C} 6$ & $107.65(12)$ \\
\hline $\mathrm{C} 8-\mathrm{C} 7-\mathrm{C} 6$ & $119.24(16)$ & & \\
\hline $\mathrm{N} 2-\mathrm{C} 1-\mathrm{C} 2-\mathrm{N} 1$ & $0.2(2)$ & $\mathrm{C} 1-\mathrm{C} 2-\mathrm{N} 1-\mathrm{C} 3$ & $-0.16(19)$ \\
\hline $\mathrm{N} 4-\mathrm{C} 1-\mathrm{C} 2-\mathrm{N} 1$ & $178.92(15)$ & $\mathrm{N} 1-\mathrm{C} 3-\mathrm{N} 2-\mathrm{C} 1$ & $0.01(19)$ \\
\hline $\mathrm{N} 1-\mathrm{C} 3-\mathrm{C} 5-\mathrm{N} 3$ & $-8.6(3)$ & $\mathrm{C} 5-\mathrm{C} 3-\mathrm{N} 2-\mathrm{C} 1$ & $176.86(15)$ \\
\hline $\mathrm{N} 2-\mathrm{C} 3-\mathrm{C} 5-\mathrm{N} 3$ & $175.00(15)$ & $\mathrm{N} 1-\mathrm{C} 3-\mathrm{N} 2-\mathrm{C} 4$ & $174.49(15)$ \\
\hline $\mathrm{O} 1-\mathrm{C} 6-\mathrm{C} 7-\mathrm{C} 8$ & $138.31(16)$ & $\mathrm{C} 5-\mathrm{C} 3-\mathrm{N} 2-\mathrm{C} 4$ & $-8.7(2)$ \\
\hline $\mathrm{O} 1-\mathrm{C} 6-\mathrm{C} 7-\mathrm{C} 12$ & $-45.5(2)$ & $\mathrm{C} 2-\mathrm{C} 1-\mathrm{N} 2-\mathrm{C} 3$ & $-0.11(18)$ \\
\hline $\mathrm{C} 12-\mathrm{C} 7-\mathrm{C} 8-\mathrm{C} 9$ & $-2.0(3)$ & $\mathrm{N} 4-\mathrm{C} 1-\mathrm{N} 2-\mathrm{C} 3$ & $-178.90(15)$ \\
\hline $\mathrm{C} 6-\mathrm{C} 7-\mathrm{C} 8-\mathrm{C} 9$ & $174.21(16)$ & $\mathrm{C} 2-\mathrm{C} 1-\mathrm{N} 2-\mathrm{C} 4$ & $-174.33(16)$ \\
\hline $\mathrm{C} 7-\mathrm{C} 8-\mathrm{C} 9-\mathrm{C} 10$ & $1.4(3)$ & $\mathrm{N} 4-\mathrm{C} 1-\mathrm{N} 2-\mathrm{C} 4$ & $6.9(3)$ \\
\hline $\mathrm{C} 8-\mathrm{C} 9-\mathrm{C} 10-\mathrm{C} 11$ & $0.3(3)$ & $\mathrm{C} 3-\mathrm{C} 5-\mathrm{N} 3-\mathrm{O} 1$ & $175.55(14)$ \\
\hline $\mathrm{C} 9-\mathrm{C} 10-\mathrm{C} 11-\mathrm{C} 12$ & $-1.5(3)$ & $\mathrm{C} 2-\mathrm{C} 1-\mathrm{N} 4-\mathrm{O} 2$ & $-171.21(18)$ \\
\hline $\mathrm{C} 10-\mathrm{C} 11-\mathrm{C} 12-\mathrm{C} 7$ & $0.9(3)$ & $\mathrm{N} 2-\mathrm{C} 1-\mathrm{N} 4-\mathrm{O} 2$ & $7.4(3)$ \\
\hline $\mathrm{C} 8-\mathrm{C} 7-\mathrm{C} 12-\mathrm{C} 11$ & $0.9(3)$ & $\mathrm{C} 2-\mathrm{C} 1-\mathrm{N} 4-\mathrm{O} 3$ & $8.3(3)$ \\
\hline $\mathrm{C} 6-\mathrm{C} 7-\mathrm{C} 12-\mathrm{C} 11$ & $-175.28(16)$ & $\mathrm{N} 2-\mathrm{C} 1-\mathrm{N} 4-\mathrm{O} 3$ & $-173.14(16)$ \\
\hline $\mathrm{N} 2-\mathrm{C} 3-\mathrm{N} 1-\mathrm{C} 2$ & 0.09 (19) & $\mathrm{C} 5-\mathrm{N} 3-\mathrm{O} 1-\mathrm{C} 6$ & $-172.50(15)$ \\
\hline $\mathrm{C} 5-\mathrm{C} 3-\mathrm{N} 1-\mathrm{C} 2$ & $-176.59(16)$ & $\mathrm{C} 7-\mathrm{C} 6-\mathrm{O} 1-\mathrm{N} 3$ & $172.62(14)$ \\
\hline
\end{tabular}

Hydrogen-bond geometry $\left(\AA,{ }^{\circ}\right)$

\begin{tabular}{lllll}
\hline$D-\mathrm{H} \cdots A$ & $D-\mathrm{H}$ & $\mathrm{H} \cdots A$ & $D \cdots A$ & $D-\mathrm{H} \cdots A$ \\
\hline $\mathrm{C} 4-\mathrm{H} 4 B \cdots \mathrm{N} 3^{\mathrm{i}}$ & 0.98 & 2.51 & $3.466(2)$ & 165 \\
$\mathrm{C} 5-\mathrm{H} 5 \cdots 3^{\mathrm{ii}}$ & 0.95 & 2.65 & $3.175(2)$ & 115 \\
\hline
\end{tabular}

Symmetry codes: (i) $-x, y+1 / 2,-z+1 / 2$; (ii) $x,-y+1 / 2, z+1 / 2$.

(E)-1-Methyl-5-nitro-1H-imidazole-2-carbaldehyde $O$-(4-fluorobenzyl) oxime (II)

Crystal data

$\mathrm{C}_{12} \mathrm{H}_{11} \mathrm{FN}_{4} \mathrm{O}_{3}$

$M_{r}=278.25$

Monoclinic, $P 2_{1} / n$

$a=7.5484(2) \AA$

$b=12.6442(4) \AA$

$c=13.4150(9) \AA$

$\beta=102.988(7)^{\circ}$

$$
\begin{aligned}
& V=1247.62(10) \AA^{3} \\
& Z=4 \\
& F(000)=576 \\
& D_{\mathrm{x}}=1.481 \mathrm{Mg} \mathrm{m}^{-3}
\end{aligned}
$$

Mo $K \alpha$ radiation, $\lambda=0.71073 \AA$

Cell parameters from 7472 reflections

$\theta=3.1-27.5^{\circ}$ 
$\mu=0.12 \mathrm{~mm}^{-1}$
$T=120 \mathrm{~K}$

Data collection

Rigaku Saturn724+ CCD diffractometer

$\omega$ scans

Absorption correction: multi-scan

(FS_ABSCOR; Rigaku, 2013)

$T_{\min }=\overline{0} .802, T_{\max }=1.000$

8522 measured reflections

\section{Refinement}

Refinement on $F^{2}$

Least-squares matrix: full

$R\left[F^{2}>2 \sigma\left(F^{2}\right)\right]=0.034$

$w R\left(F^{2}\right)=0.094$

$S=1.09$

2848 reflections

182 parameters

0 restraints
Block, orange

$0.19 \times 0.13 \times 0.10 \mathrm{~mm}$

2848 independent reflections

2292 reflections with $I>2 \sigma(I)$

$R_{\text {int }}=0.021$

$\theta_{\text {max }}=27.5^{\circ}, \theta_{\text {min }}=3.1^{\circ}$

$h=-9 \rightarrow 9$

$k=-14 \rightarrow 16$

$l=-14 \rightarrow 17$

Primary atom site location: structure-invariant direct methods

Hydrogen site location: inferred from neighbouring sites

$\mathrm{H}$-atom parameters constrained

$w=1 /\left[\sigma^{2}\left(F_{\mathrm{o}}^{2}\right)+(0.0501 P)^{2}+0.1808 P\right]$

where $P=\left(F_{\mathrm{o}}^{2}+2 F_{\mathrm{c}}^{2}\right) / 3$

$(\Delta / \sigma)_{\max }<0.001$

$\Delta \rho_{\max }=0.29$ e $\AA^{-3}$

$\Delta \rho_{\min }=-0.18$ e $\AA^{-3}$

Special details

Geometry. All esds (except the esd in the dihedral angle between two 1.s. planes) are estimated using the full covariance matrix. The cell esds are taken into account individually in the estimation of esds in distances, angles and torsion angles; correlations between esds in cell parameters are only used when they are defined by crystal symmetry. An approximate (isotropic) treatment of cell esds is used for estimating esds involving 1.s. planes.

Fractional atomic coordinates and isotropic or equivalent isotropic displacement parameters $\left(\AA^{2}\right)$

\begin{tabular}{lllll}
\hline & $x$ & $y$ & $z$ & $U_{\text {iso }} * / U_{\text {eq }}$ \\
\hline C1 & $0.40044(15)$ & $0.59196(9)$ & $0.16775(9)$ & $0.0177(2)$ \\
C2 & $0.38559(16)$ & $0.48697(9)$ & $0.14129(9)$ & $0.0192(3)$ \\
H2 & 0.3923 & 0.4590 & 0.0765 & $0.023^{*}$ \\
C3 & $0.35720(15)$ & $0.49975(9)$ & $0.29626(9)$ & $0.0160(2)$ \\
C4 & $0.40828(17)$ & $0.69554(9)$ & $0.33328(9)$ & $0.0213(3)$ \\
H4A & 0.3204 & 0.7499 & 0.3025 & $0.032^{*}$ \\
H4B & 0.5320 & 0.7228 & 0.3401 & $0.032^{*}$ \\
H4C & 0.3892 & 0.6769 & 0.4010 & $0.032^{*}$ \\
C5 & $0.32784(15)$ & $0.46335(9)$ & $0.39394(9)$ & $0.0176(2)$ \\
H5 & 0.3457 & 0.3906 & 0.4109 & $0.021^{*}$ \\
C6 & $0.18665(15)$ & $0.54029(9)$ & $0.60895(9)$ & $0.0185(2)$ \\
H6A & 0.0718 & 0.5716 & 0.5697 & $0.022^{*}$ \\
H6B & 0.2737 & 0.5984 & 0.6330 & $0.022^{*}$ \\
C7 & $0.15188(14)$ & $0.47943(9)$ & $0.69873(9)$ & $0.0175(2)$ \\
C8 & $0.10500(15)$ & $0.37210(9)$ & $0.69078(9)$ & $0.0201(3)$ \\
H8 & 0.1018 & 0.3354 & 0.6286 & $0.024^{*}$ \\
C9 & $0.06311(16)$ & $0.31892(10)$ & $0.77312(10)$ & $0.0238(3)$ \\
H9 & 0.0328 & 0.2459 & 0.7684 & $0.029 *$
\end{tabular}




\begin{tabular}{lllll} 
C10 & $0.06662(16)$ & $0.37454(11)$ & $0.86169(10)$ & $0.0249(3)$ \\
C11 & $0.11210(17)$ & $0.48020(11)$ & $0.87283(9)$ & $0.0250(3)$ \\
H11 & 0.1132 & 0.5165 & 0.9350 & $0.030^{*}$ \\
C12 & $0.15620(16)$ & $0.53179(10)$ & $0.79027(9)$ & $0.0210(3)$ \\
H12 & 0.1900 & 0.6043 & 0.7965 & $0.025^{*}$ \\
N1 & $0.36001(13)$ & $0.42969(8)$ & $0.22186(7)$ & $0.0187(2)$ \\
N2 & $0.38326(12)$ & $0.60100(7)$ & $0.26758(7)$ & $0.0160(2)$ \\
N3 & $0.27904(12)$ & $0.52518(8)$ & $0.45773(7)$ & $0.0180(2)$ \\
N4 & $0.42710(14)$ & $0.67827(8)$ & $0.10515(8)$ & $0.0225(2)$ \\
O1 & $0.26029(11)$ & $0.46915(6)$ & $0.54488(6)$ & $0.01878(19)$ \\
O2 & $0.44180(14)$ & $0.76801(7)$ & $0.14148(7)$ & $0.0315(2)$ \\
O3 & $0.43216(14)$ & $0.65795(8)$ & $0.01587(7)$ & $0.0338(2)$ \\
F1 & $0.02098(11)$ & $0.32309(7)$ & $0.94206(6)$ & $0.0378(2)$ \\
\hline
\end{tabular}

Atomic displacement parameters $\left(A^{2}\right)$

\begin{tabular}{lllllll}
\hline & $U^{11}$ & $U^{22}$ & $U^{33}$ & $U^{12}$ & $U^{13}$ & $U^{23}$ \\
\hline C1 & $0.0181(5)$ & $0.0188(6)$ & $0.0166(6)$ & $0.0022(4)$ & $0.0048(4)$ & $0.0022(5)$ \\
C2 & $0.0219(6)$ & $0.0197(6)$ & $0.0162(6)$ & $0.0018(4)$ & $0.0051(4)$ & $-0.0009(5)$ \\
C3 & $0.0138(5)$ & $0.0155(5)$ & $0.0181(6)$ & $0.0007(4)$ & $0.0022(4)$ & $0.0002(5)$ \\
C4 & $0.0275(6)$ & $0.0157(6)$ & $0.0215(6)$ & $-0.0017(4)$ & $0.0071(5)$ & $-0.0037(5)$ \\
C5 & $0.0177(5)$ & $0.0164(6)$ & $0.0184(6)$ & $-0.0011(4)$ & $0.0030(4)$ & $0.0005(5)$ \\
C6 & $0.0188(5)$ & $0.0187(6)$ & $0.0185(6)$ & $0.0011(4)$ & $0.0055(4)$ & $-0.0031(5)$ \\
C7 & $0.0121(5)$ & $0.0208(6)$ & $0.0189(6)$ & $0.0023(4)$ & $0.0022(4)$ & $0.0004(5)$ \\
C8 & $0.0180(5)$ & $0.0211(6)$ & $0.0206(6)$ & $0.0016(4)$ & $0.0031(4)$ & $-0.0007(5)$ \\
C9 & $0.0214(6)$ & $0.0211(6)$ & $0.0286(7)$ & $0.0017(5)$ & $0.0049(5)$ & $0.0053(5)$ \\
C10 & $0.0212(6)$ & $0.0328(7)$ & $0.0217(6)$ & $0.0045(5)$ & $0.0067(5)$ & $0.0111(5)$ \\
C11 & $0.0237(6)$ & $0.0342(7)$ & $0.0174(6)$ & $0.0031(5)$ & $0.0053(5)$ & $-0.0008(5)$ \\
C12 & $0.0188(6)$ & $0.0221(6)$ & $0.0218(6)$ & $0.0010(4)$ & $0.0037(4)$ & $-0.0012(5)$ \\
N1 & $0.0203(5)$ & $0.0175(5)$ & $0.0181(5)$ & $0.0003(4)$ & $0.0042(4)$ & $-0.0013(4)$ \\
N2 & $0.0164(4)$ & $0.0146(5)$ & $0.0172(5)$ & $0.0012(4)$ & $0.0041(4)$ & $0.0005(4)$ \\
N3 & $0.0170(5)$ & $0.0200(5)$ & $0.0167(5)$ & $-0.0001(4)$ & $0.0032(4)$ & $0.0030(4)$ \\
N4 & $0.0237(5)$ & $0.0212(5)$ & $0.0243(6)$ & $0.0027(4)$ & $0.0092(4)$ & $0.0038(4)$ \\
O1 & $0.0230(4)$ & $0.0186(4)$ & $0.0164(4)$ & $0.0026(3)$ & $0.0077(3)$ & $0.0022(3)$ \\
O2 & $0.0468(6)$ & $0.0176(5)$ & $0.0332(6)$ & $-0.0032(4)$ & $0.0158(4)$ & $0.0013(4)$ \\
O3 & $0.0537(6)$ & $0.0307(5)$ & $0.0217(5)$ & $0.0033(4)$ & $0.0182(4)$ & $0.0037(4)$ \\
F1 & $0.0437(5)$ & $0.0446(5)$ & $0.0286(4)$ & $0.0027(4)$ & $0.0158(4)$ & $0.0162(4)$ \\
& & & & & &
\end{tabular}

Geometric parameters ( $\left.\AA,{ }^{\circ}\right)$

\begin{tabular}{llll}
\hline $\mathrm{C} 1-\mathrm{C} 2$ & $1.3721(16)$ & $\mathrm{C} 6-\mathrm{H} 6 \mathrm{~A}$ & 0.9900 \\
$\mathrm{C} 1-\mathrm{N} 2$ & $1.3789(15)$ & $\mathrm{C} 6-\mathrm{H} 6 \mathrm{~B}$ & 0.9900 \\
$\mathrm{C} 1-\mathrm{N} 4$ & $1.4187(15)$ & $\mathrm{C} 7-\mathrm{C} 12$ & $1.3890(16)$ \\
$\mathrm{C} 2-\mathrm{N} 1$ & $1.3506(15)$ & $\mathrm{C} 7-\mathrm{C} 8$ & $1.3890(17)$ \\
$\mathrm{C} 2-\mathrm{H} 2$ & 0.9500 & $\mathrm{C} 8-\mathrm{C} 9$ & 0.9500 \\
$\mathrm{C} 3-\mathrm{N} 1$ & $1.3382(15)$ & $\mathrm{C} 8-\mathrm{H} 8$ & $1.3757(18)$ \\
$\mathrm{C} 3-\mathrm{N} 2$ & $1.3636(15)$ & $\mathrm{C} 9-\mathrm{C} 10$ & 0.9500 \\
$\mathrm{C} 3-\mathrm{C} 5$ & $1.4522(16)$ & $\mathrm{C} 9-\mathrm{H} 9$ &
\end{tabular}




\begin{tabular}{|c|c|c|c|}
\hline $\mathrm{C} 4-\mathrm{N} 2$ & $1.4721(15)$ & $\mathrm{C} 10-\mathrm{F} 1$ & $1.3679(14)$ \\
\hline $\mathrm{C} 4-\mathrm{H} 4 \mathrm{~A}$ & 0.9800 & $\mathrm{C} 10-\mathrm{C} 11$ & $1.3791(19)$ \\
\hline $\mathrm{C} 4-\mathrm{H} 4 \mathrm{~B}$ & 0.9800 & $\mathrm{C} 11-\mathrm{C} 12$ & $1.3888(17)$ \\
\hline $\mathrm{C} 4-\mathrm{H} 4 \mathrm{C}$ & 0.9800 & $\mathrm{C} 11-\mathrm{H} 11$ & 0.9500 \\
\hline $\mathrm{C} 5-\mathrm{N} 3$ & $1.2731(15)$ & $\mathrm{C} 12-\mathrm{H} 12$ & 0.9500 \\
\hline $\mathrm{C} 5-\mathrm{H} 5$ & 0.9500 & $\mathrm{~N} 3-\mathrm{O} 1$ & $1.4012(12)$ \\
\hline $\mathrm{C} 6-\mathrm{O} 1$ & $1.4393(13)$ & $\mathrm{N} 4-\mathrm{O} 2$ & $1.2301(14)$ \\
\hline $\mathrm{C} 6-\mathrm{C} 7$ & $1.5013(16)$ & $\mathrm{N} 4-\mathrm{O} 3$ & $1.2339(14)$ \\
\hline $\mathrm{C} 2-\mathrm{C} 1-\mathrm{N} 2$ & $108.14(10)$ & $\mathrm{C} 8-\mathrm{C} 7-\mathrm{C} 6$ & $121.49(11)$ \\
\hline $\mathrm{C} 2-\mathrm{C} 1-\mathrm{N} 4$ & $127.25(11)$ & $\mathrm{C} 9-\mathrm{C} 8-\mathrm{C} 7$ & $120.48(11)$ \\
\hline $\mathrm{N} 2-\mathrm{C} 1-\mathrm{N} 4$ & $124.60(10)$ & $\mathrm{C} 9-\mathrm{C} 8-\mathrm{H} 8$ & 119.8 \\
\hline $\mathrm{N} 1-\mathrm{C} 2-\mathrm{C} 1$ & $109.26(10)$ & $\mathrm{C} 7-\mathrm{C} 8-\mathrm{H} 8$ & 119.8 \\
\hline $\mathrm{N} 1-\mathrm{C} 2-\mathrm{H} 2$ & 125.4 & $\mathrm{C} 10-\mathrm{C} 9-\mathrm{C} 8$ & $118.41(12)$ \\
\hline $\mathrm{C} 1-\mathrm{C} 2-\mathrm{H} 2$ & 125.4 & $\mathrm{C} 10-\mathrm{C} 9-\mathrm{H} 9$ & 120.8 \\
\hline $\mathrm{N} 1-\mathrm{C} 3-\mathrm{N} 2$ & $112.56(10)$ & $\mathrm{C} 8-\mathrm{C} 9-\mathrm{H} 9$ & 120.8 \\
\hline $\mathrm{N} 1-\mathrm{C} 3-\mathrm{C} 5$ & $119.63(10)$ & $\mathrm{F} 1-\mathrm{C} 10-\mathrm{C} 9$ & $118.57(12)$ \\
\hline $\mathrm{N} 2-\mathrm{C} 3-\mathrm{C} 5$ & $127.81(10)$ & $\mathrm{F} 1-\mathrm{C} 10-\mathrm{C} 11$ & $118.42(12)$ \\
\hline $\mathrm{N} 2-\mathrm{C} 4-\mathrm{H} 4 \mathrm{~A}$ & 109.5 & $\mathrm{C} 9-\mathrm{C} 10-\mathrm{C} 11$ & $123.01(12)$ \\
\hline $\mathrm{N} 2-\mathrm{C} 4-\mathrm{H} 4 \mathrm{~B}$ & 109.5 & $\mathrm{C} 10-\mathrm{C} 11-\mathrm{C} 12$ & $117.84(12)$ \\
\hline $\mathrm{H} 4 \mathrm{~A}-\mathrm{C} 4-\mathrm{H} 4 \mathrm{~B}$ & 109.5 & $\mathrm{C} 10-\mathrm{C} 11-\mathrm{H} 11$ & 121.1 \\
\hline $\mathrm{N} 2-\mathrm{C} 4-\mathrm{H} 4 \mathrm{C}$ & 109.5 & $\mathrm{C} 12-\mathrm{C} 11-\mathrm{H} 11$ & 121.1 \\
\hline $\mathrm{H} 4 \mathrm{~A}-\mathrm{C} 4-\mathrm{H} 4 \mathrm{C}$ & 109.5 & $\mathrm{C} 11-\mathrm{C} 12-\mathrm{C} 7$ & $121.26(11)$ \\
\hline $\mathrm{H} 4 \mathrm{~B}-\mathrm{C} 4-\mathrm{H} 4 \mathrm{C}$ & 109.5 & $\mathrm{C} 11-\mathrm{C} 12-\mathrm{H} 12$ & 119.4 \\
\hline $\mathrm{N} 3-\mathrm{C} 5-\mathrm{C} 3$ & $122.53(11)$ & $\mathrm{C} 7-\mathrm{C} 12-\mathrm{H} 12$ & 119.4 \\
\hline $\mathrm{N} 3-\mathrm{C} 5-\mathrm{H} 5$ & 118.7 & $\mathrm{C} 3-\mathrm{N} 1-\mathrm{C} 2$ & $105.71(10)$ \\
\hline $\mathrm{C} 3-\mathrm{C} 5-\mathrm{H} 5$ & 118.7 & $\mathrm{C} 3-\mathrm{N} 2-\mathrm{C} 1$ & $104.31(9)$ \\
\hline $\mathrm{O} 1-\mathrm{C} 6-\mathrm{C} 7$ & $108.62(9)$ & $\mathrm{C} 3-\mathrm{N} 2-\mathrm{C} 4$ & $126.92(10)$ \\
\hline $\mathrm{O} 1-\mathrm{C} 6-\mathrm{H} 6 \mathrm{~A}$ & 110.0 & $\mathrm{C} 1-\mathrm{N} 2-\mathrm{C} 4$ & $128.42(10)$ \\
\hline $\mathrm{C} 7-\mathrm{C} 6-\mathrm{H} 6 \mathrm{~A}$ & 110.0 & $\mathrm{C} 5-\mathrm{N} 3-\mathrm{O} 1$ & $110.43(9)$ \\
\hline $\mathrm{O} 1-\mathrm{C} 6-\mathrm{H} 6 \mathrm{~B}$ & 110.0 & $\mathrm{O} 2-\mathrm{N} 4-\mathrm{O} 3$ & $123.85(11)$ \\
\hline $\mathrm{C} 7-\mathrm{C} 6-\mathrm{H} 6 \mathrm{~B}$ & 110.0 & $\mathrm{O} 2-\mathrm{N} 4-\mathrm{C} 1$ & $119.16(10)$ \\
\hline $\mathrm{H} 6 \mathrm{~A}-\mathrm{C} 6-\mathrm{H} 6 \mathrm{~B}$ & 108.3 & $\mathrm{O} 3-\mathrm{N} 4-\mathrm{C} 1$ & $116.99(10)$ \\
\hline $\mathrm{C} 12-\mathrm{C} 7-\mathrm{C} 8$ & $118.98(11)$ & $\mathrm{N} 3-\mathrm{O} 1-\mathrm{C} 6$ & $107.89(8)$ \\
\hline $\mathrm{C} 12-\mathrm{C} 7-\mathrm{C} 6$ & $119.43(10)$ & & \\
\hline $\mathrm{N} 2-\mathrm{C} 1-\mathrm{C} 2-\mathrm{N} 1$ & $-0.16(13)$ & $\mathrm{C} 5-\mathrm{C} 3-\mathrm{N} 1-\mathrm{C} 2$ & $178.73(10)$ \\
\hline $\mathrm{N} 4-\mathrm{C} 1-\mathrm{C} 2-\mathrm{N} 1$ & $-179.62(11)$ & $\mathrm{C} 1-\mathrm{C} 2-\mathrm{N} 1-\mathrm{C} 3$ & $0.72(13)$ \\
\hline $\mathrm{N} 1-\mathrm{C} 3-\mathrm{C} 5-\mathrm{N} 3$ & $-162.10(10)$ & $\mathrm{N} 1-\mathrm{C} 3-\mathrm{N} 2-\mathrm{C} 1$ & $0.95(12)$ \\
\hline $\mathrm{N} 2-\mathrm{C} 3-\mathrm{C} 5-\mathrm{N} 3$ & $17.64(18)$ & $\mathrm{C} 5-\mathrm{C} 3-\mathrm{N} 2-\mathrm{C} 1$ & $-178.81(11)$ \\
\hline $\mathrm{O} 1-\mathrm{C} 6-\mathrm{C} 7-\mathrm{C} 12$ & $152.70(10)$ & $\mathrm{N} 1-\mathrm{C} 3-\mathrm{N} 2-\mathrm{C} 4$ & $-172.71(10)$ \\
\hline $\mathrm{O} 1-\mathrm{C} 6-\mathrm{C} 7-\mathrm{C} 8$ & $-30.95(14)$ & $\mathrm{C} 5-\mathrm{C} 3-\mathrm{N} 2-\mathrm{C} 4$ & $7.53(18)$ \\
\hline $\mathrm{C} 12-\mathrm{C} 7-\mathrm{C} 8-\mathrm{C} 9$ & $0.13(16)$ & $\mathrm{C} 2-\mathrm{C} 1-\mathrm{N} 2-\mathrm{C} 3$ & $-0.46(12)$ \\
\hline $\mathrm{C} 6-\mathrm{C} 7-\mathrm{C} 8-\mathrm{C} 9$ & $-176.23(10)$ & $\mathrm{N} 4-\mathrm{C} 1-\mathrm{N} 2-\mathrm{C} 3$ & $179.02(10)$ \\
\hline $\mathrm{C} 7-\mathrm{C} 8-\mathrm{C} 9-\mathrm{C} 10$ & $0.82(17)$ & $\mathrm{C} 2-\mathrm{C} 1-\mathrm{N} 2-\mathrm{C} 4$ & $173.07(10)$ \\
\hline $\mathrm{C} 8-\mathrm{C} 9-\mathrm{C} 10-\mathrm{F} 1$ & $178.37(10)$ & $\mathrm{N} 4-\mathrm{C} 1-\mathrm{N} 2-\mathrm{C} 4$ & $-7.45(18)$ \\
\hline $\mathrm{C} 8-\mathrm{C} 9-\mathrm{C} 10-\mathrm{C} 11$ & $-0.87(18)$ & $\mathrm{C} 3-\mathrm{C} 5-\mathrm{N} 3-\mathrm{O} 1$ & $179.79(9)$ \\
\hline $\mathrm{F} 1-\mathrm{C} 10-\mathrm{C} 11-\mathrm{C} 12$ & $-179.30(10)$ & $\mathrm{C} 2-\mathrm{C} 1-\mathrm{N} 4-\mathrm{O} 2$ & $-178.34(11)$ \\
\hline
\end{tabular}




$\begin{array}{llll}\mathrm{C} 9-\mathrm{C} 10-\mathrm{C} 11-\mathrm{C} 12 & -0.05(18) & \mathrm{N} 2-\mathrm{C} 1-\mathrm{N} 4-\mathrm{O} 2 & 2.29(17) \\ \mathrm{C} 10-\mathrm{C} 11-\mathrm{C} 12-\mathrm{C} 7 & 1.06(18) & \mathrm{C} 2-\mathrm{C} 1-\mathrm{N} 4-\mathrm{O} 3 & 2.41(18) \\ \mathrm{C} 8-\mathrm{C} 7-\mathrm{C} 12-\mathrm{C} 11 & -1.10(17) & \mathrm{N} 2-\mathrm{C} 1-\mathrm{N} 4-\mathrm{O} 3 & -176.96(11) \\ \mathrm{C} 6-\mathrm{C} 7-\mathrm{C} 12-\mathrm{C} 11 & 175.34(10) & \mathrm{C} 5-\mathrm{N} 3-\mathrm{O} 1-\mathrm{C} 6 & -173.96(9) \\ \mathrm{N} 2-\mathrm{C} 3-\mathrm{N} 1-\mathrm{C} 2 & -1.05(13) & \mathrm{C} 7-\mathrm{C} 6-\mathrm{O} 1-\mathrm{N} 3 & 175.61(8)\end{array}$

Hydrogen-bond geometry $\left(\AA,{ }^{\circ}\right)$

\begin{tabular}{lllll}
\hline$D-\mathrm{H} \cdots A$ & $D-\mathrm{H}$ & $\mathrm{H} \cdots A$ & $D \cdots A$ & $D-\mathrm{H} \cdots A$ \\
\hline $\mathrm{C} 4-\mathrm{H} 4 C \cdots \mathrm{N} 3$ & 0.98 & 2.29 & $3.0184(15)$ & 131 \\
$\mathrm{C} 4-\mathrm{H} 4 A \cdots \mathrm{N} 1^{\mathrm{i}}$ & 0.98 & 2.63 & $3.5693(16)$ & 160 \\
$\mathrm{C} 9-\mathrm{H} 9 \cdots \mathrm{N} 1^{\mathrm{ii}}$ & 0.95 & 2.58 & $3.4973(16)$ & 163 \\
$\mathrm{C} 2-\mathrm{H} 2 \cdots 3^{\mathrm{iii}}$ & 0.95 & 2.49 & $3.3165(15)$ & 145 \\
$\mathrm{C} 5-\mathrm{H} 5 \cdots 2^{\mathrm{iv}}$ & 0.95 & 2.63 & $3.1676(14)$ & 116 \\
$\mathrm{C} 6-\mathrm{H} 6 A \cdots \mathrm{O} 2^{\mathrm{v}}$ & 0.99 & 2.54 & $3.1376(14)$ & 119 \\
$\mathrm{C} 4-\mathrm{H} 4 C \cdots \mathrm{F} 1^{\mathrm{vi}}$ & 0.98 & 2.77 & $3.353(2)$ & 119
\end{tabular}

Symmetry codes: (i) $-x+1 / 2, y+1 / 2,-z+1 / 2$; (ii) $x-1 / 2,-y+1 / 2, z+1 / 2$; (iii) $-x+1,-y+1,-z$; (iv) $-x+1 / 2, y-1 / 2,-z+1 / 2$; (v) $x-1 / 2,-y+3 / 2, z+1 / 2$; (vi) $-x+1 / 2, y+1 / 2,-z+3 / 2$.

(E)-1-Methyl-5-nitro-1H-imidazole-2-carbaldehyde $O$-(4-bromobenzyl) oxime (III)

Crystal data

$\mathrm{C}_{12} \mathrm{H}_{11} \mathrm{BrN}_{4} \mathrm{O}_{3}$

$M_{r}=339.16$

Monoclinic, $P 2_{1} / n$

$a=7.6024(2) \AA$

$b=12.7526(3) \AA$

$c=13.8954(5) \AA$

$\beta=104.869(2)^{\circ}$

$V=1302.05(7) \AA^{3}$

$Z=4$

\section{Data collection}

Rigaku Mercury CCD diffractometer

$\omega$ scans

Absorption correction: multi-scan

(FS_ABSCOR; Rigaku, 2013)

$T_{\min }=0.438, T_{\max }=1.000$

16791 measured reflections

\section{Refinement}

Refinement on $F^{2}$

Least-squares matrix: full

$R\left[F^{2}>2 \sigma\left(F^{2}\right)\right]=0.051$

$w R\left(F^{2}\right)=0.140$

$S=1.11$

2974 reflections

182 parameters

0 restraints
$F(000)=680$

$D_{\mathrm{x}}=1.730 \mathrm{Mg} \mathrm{m}^{-3}$

Mo $K \alpha$ radiation, $\lambda=0.71073 \AA$

Cell parameters from 6837 reflections

$\theta=2.2-27.5^{\circ}$

$\mu=3.17 \mathrm{~mm}^{-1}$

$T=120 \mathrm{~K}$

Block, yellow

$0.66 \times 0.52 \times 0.24 \mathrm{~mm}$

2974 independent reflections

2835 reflections with $I>2 \sigma(I)$

$R_{\text {int }}=0.065$

$\theta_{\max }=27.5^{\circ}, \theta_{\min }=2.2^{\circ}$

$h=-8 \rightarrow 9$

$k=-16 \rightarrow 16$

$l=-18 \rightarrow 16$

Primary atom site location: structure-invariant direct methods

Hydrogen site location: inferred from neighbouring sites

$\mathrm{H}$-atom parameters constrained

$w=1 /\left[\sigma^{2}\left(F_{\mathrm{o}}^{2}\right)+(0.0837 P)^{2}+1.3179 P\right]$

where $P=\left(F_{\mathrm{o}}{ }^{2}+2 F_{\mathrm{c}}{ }^{2}\right) / 3$

$(\Delta / \sigma)_{\max }=0.001$

$\Delta \rho_{\max }=1.78$ e $\AA^{-3}$

$\Delta \rho_{\min }=-1.03$ e $\AA^{-3}$ 


\section{Special details}

Geometry. All esds (except the esd in the dihedral angle between two 1.s. planes) are estimated using the full covariance matrix. The cell esds are taken into account individually in the estimation of esds in distances, angles and torsion angles; correlations between esds in cell parameters are only used when they are defined by crystal symmetry. An approximate (isotropic) treatment of cell esds is used for estimating esds involving l.s. planes.

Fractional atomic coordinates and isotropic or equivalent isotropic displacement parameters $\left(\hat{A}^{2}\right)$

\begin{tabular}{|c|c|c|c|c|}
\hline & $x$ & $y$ & $z$ & $U_{\text {iso }} * / U_{\text {eq }}$ \\
\hline $\mathrm{C} 1$ & $0.4010(4)$ & $0.5860(2)$ & $0.1628(2)$ & $0.0210(6)$ \\
\hline $\mathrm{C} 2$ & $0.3825(4)$ & $0.4812(2)$ & $0.1385(2)$ & $0.0227(6)$ \\
\hline $\mathrm{H} 2$ & 0.3902 & 0.4517 & 0.0769 & $0.027 *$ \\
\hline $\mathrm{C} 3$ & $0.3498(4)$ & $0.4984(2)$ & $0.2868(2)$ & $0.0201(6)$ \\
\hline $\mathrm{C} 4$ & $0.3974(5)$ & $0.6933(2)$ & $0.3184(3)$ & $0.0281(7)$ \\
\hline $\mathrm{H} 4 \mathrm{~A}$ & 0.3108 & 0.7457 & 0.2827 & $0.042 *$ \\
\hline $\mathrm{H} 4 \mathrm{~B}$ & 0.5214 & 0.7210 & 0.3308 & $0.042 *$ \\
\hline $\mathrm{H} 4 \mathrm{C}$ & 0.3704 & 0.6770 & 0.3821 & $0.042 *$ \\
\hline $\mathrm{C} 5$ & $0.3150(4)$ & $0.4652(2)$ & $0.3803(2)$ & $0.0221(6)$ \\
\hline H5 & 0.3120 & 0.3922 & 0.3932 & $0.026^{*}$ \\
\hline C6 & $0.2011(4)$ & $0.5460(2)$ & $0.5916(2)$ & $0.0227(6)$ \\
\hline H6A & 0.0894 & 0.5833 & 0.5554 & $0.027^{*}$ \\
\hline H6B & 0.2982 & 0.5986 & 0.6159 & $0.027^{*}$ \\
\hline $\mathrm{C} 7$ & $0.1655(4)$ & $0.4863(2)$ & $0.6778(2)$ & $0.0203(6)$ \\
\hline $\mathrm{C} 8$ & $0.1233(4)$ & $0.3798(2)$ & $0.6718(2)$ & $0.0229(6)$ \\
\hline $\mathrm{H} 8$ & 0.1207 & 0.3422 & 0.6124 & $0.027^{*}$ \\
\hline C9 & $0.0849(4)$ & $0.3284(2)$ & $0.7522(2)$ & $0.0237(6)$ \\
\hline H9 & 0.0561 & 0.2558 & 0.7481 & $0.028^{*}$ \\
\hline $\mathrm{C} 10$ & $0.0890(4)$ & $0.3840(2)$ & $0.8382(2)$ & $0.0224(6)$ \\
\hline C11 & $0.1292(4)$ & $0.4900(2)$ & $0.8463(2)$ & $0.0232(6)$ \\
\hline H11 & 0.1300 & 0.5275 & 0.9055 & $0.028^{*}$ \\
\hline $\mathrm{C} 12$ & $0.1683(4)$ & $0.5400(2)$ & $0.7656(2)$ & $0.0232(6)$ \\
\hline H12 & 0.1977 & 0.6126 & 0.7702 & $0.028^{*}$ \\
\hline N1 & $0.3517(4)$ & $0.42704(18)$ & $0.21619(19)$ & $0.0233(5)$ \\
\hline N2 & $0.3819(3)$ & $0.59752(17)$ & $0.25814(18)$ & $0.0191(5)$ \\
\hline N3 & $0.2882(3)$ & $0.52867(18)$ & $0.44583(19)$ & $0.0219(5)$ \\
\hline N4 & $0.4338(4)$ & $0.6687(2)$ & $0.1020(2)$ & $0.0234(5)$ \\
\hline $\mathrm{O} 1$ & $0.2561(3)$ & $0.47297(15)$ & $0.52654(16)$ & $0.0239(5)$ \\
\hline $\mathrm{O} 2$ & $0.4605(4)$ & $0.75804(16)$ & 0.13734 (19) & $0.0325(5)$ \\
\hline $\mathrm{O} 3$ & $0.4324(4)$ & 0.64727 (19) & $0.01529(19)$ & $0.0343(6)$ \\
\hline $\mathrm{Br} 1$ & $0.04121(4)$ & $0.31299(2)$ & $0.94938(2)$ & $0.02821(16)$ \\
\hline
\end{tabular}

Atomic displacement parameters $\left(\AA^{2}\right)$

\begin{tabular}{lllllll}
\hline & $U^{11}$ & $U^{22}$ & $U^{33}$ & $U^{12}$ & $U^{13}$ & $U^{23}$ \\
\hline C1 & $0.0257(14)$ & $0.0171(12)$ & $0.0208(14)$ & $0.0008(10)$ & $0.0071(11)$ & $-0.0021(10)$ \\
C2 & $0.0304(15)$ & $0.0168(12)$ & $0.0214(15)$ & $0.0020(10)$ & $0.0076(12)$ & $-0.0035(10)$ \\
C3 & $0.0216(13)$ & $0.0147(11)$ & $0.0239(15)$ & $0.0002(10)$ & $0.0058(11)$ & $-0.0007(10)$ \\
C4 & $0.0431(19)$ & $0.0137(13)$ & $0.0295(18)$ & $-0.0020(11)$ & $0.0132(15)$ & $-0.0045(10)$
\end{tabular}


supporting information

$\begin{array}{lllllll}\text { C5 } & 0.0241(14) & 0.0144(11) & 0.0284(16) & 0.0010(10) & 0.0078(12) & 0.0019(10) \\ \text { C6 } & 0.0284(15) & 0.0165(12) & 0.0237(15) & 0.0008(10) & 0.0079(12) & 0.0004(10) \\ \text { C7 } & 0.0198(13) & 0.0173(12) & 0.0231(15) & 0.0017(10) & 0.0042(11) & 0.0025(10) \\ \text { C8 } & 0.0259(14) & 0.0168(12) & 0.0256(15) & 0.0013(10) & 0.0058(11) & -0.0016(10) \\ \text { C9 } & 0.0279(15) & 0.0140(11) & 0.0291(17) & 0.0004(10) & 0.0072(12) & 0.0008(11) \\ \text { C10 } & 0.0218(14) & 0.0196(13) & 0.0262(15) & 0.0019(10) & 0.0069(11) & 0.0052(10) \\ \text { C11 } & 0.0275(15) & 0.0202(13) & 0.0221(15) & 0.0010(11) & 0.0067(12) & -0.0016(10) \\ \text { C12 } & 0.0290(15) & 0.0141(12) & 0.0269(16) & -0.0015(10) & 0.0082(12) & 0.0000(10) \\ \text { N1 } & 0.0305(13) & 0.0144(10) & 0.0255(13) & -0.0002(9) & 0.0083(10) & -0.0035(9) \\ \text { N2 } & 0.0261(12) & 0.0126(10) & 0.0195(12) & -0.0001(8) & 0.0071(9) & -0.0015(9) \\ \text { N3 } & 0.0263(12) & 0.0176(10) & 0.0227(13) & -0.0007(9) & 0.0079(10) & 0.0041(9) \\ \text { N4 } & 0.0268(13) & 0.0188(11) & 0.0257(14) & 0.0005(9) & 0.0090(11) & 0.0011(10) \\ \text { O1 } & 0.0328(12) & 0.0170(9) & 0.0253(12) & 0.0019(8) & 0.0137(9) & 0.0027(8) \\ \text { O2 } & 0.0485(14) & 0.0161(10) & 0.0357(13) & -0.0055(9) & 0.0158(11) & -0.0015(9) \\ \text { O3 } & 0.0536(15) & 0.0290(12) & 0.0251(13) & 0.0010(11) & 0.0190(11) & -0.0004(10) \\ \text { Br1 } & 0.0366(2) & 0.0231(2) & 0.0267(2) & 0.00051(10) & 0.01133(17) & 0.00691(10) \\ & & & & & \end{array}$

Geometric parameters $\left(\AA,{ }^{\circ}\right)$

\begin{tabular}{llll}
\hline $\mathrm{C} 1-\mathrm{C} 2$ & $1.377(4)$ & $\mathrm{C} 6-\mathrm{H} 6 \mathrm{~A}$ & 0.9900 \\
$\mathrm{C} 1-\mathrm{N} 2$ & $1.378(4)$ & $\mathrm{C} 6-\mathrm{H} 6 \mathrm{~B}$ & 0.9900 \\
$\mathrm{C} 1-\mathrm{N} 4$ & $1.413(4)$ & $\mathrm{C} 7-\mathrm{C} 8$ & $1.393(4)$ \\
$\mathrm{C} 2-\mathrm{N} 1$ & $1.352(4)$ & $\mathrm{C} 7-\mathrm{C} 12$ & $1.394(4)$ \\
$\mathrm{C} 2-\mathrm{H} 2$ & 0.9500 & $\mathrm{C} 8-\mathrm{C} 9$ & $1.389(4)$ \\
$\mathrm{C} 3-\mathrm{N} 1$ & $1.341(4)$ & $\mathrm{C} 8-\mathrm{H} 8$ & 0.9500 \\
$\mathrm{C} 3-\mathrm{N} 2$ & $1.366(3)$ & $\mathrm{C} 9-\mathrm{C} 10$ & $1.383(4)$ \\
$\mathrm{C} 3-\mathrm{C} 5$ & $1.453(4)$ & $\mathrm{C} 9-\mathrm{H} 9$ & 0.9500 \\
$\mathrm{C} 4-\mathrm{N} 2$ & $1.468(3)$ & $\mathrm{C} 10-\mathrm{C} 11$ & $1.384(4)$ \\
$\mathrm{C} 4-\mathrm{H} 4 \mathrm{~A}$ & 0.9800 & $\mathrm{C} 10-\mathrm{B} 1$ & $1.905(3)$ \\
$\mathrm{C} 4-\mathrm{H} 4 \mathrm{~B}$ & 0.9800 & $\mathrm{C} 11-\mathrm{C} 12$ & $1.387(4)$ \\
$\mathrm{C} 4-\mathrm{H} 4 \mathrm{C}$ & 0.9800 & $\mathrm{C} 11-\mathrm{H} 11$ & 0.9500 \\
$\mathrm{C} 5-\mathrm{N} 3$ & $1.273(4)$ & $\mathrm{C} 12-\mathrm{H} 12$ & 0.9500 \\
$\mathrm{C} 5-\mathrm{H} 5$ & 0.9500 & $\mathrm{~N} 4-\mathrm{O} 1$ & $1.401(3)$ \\
$\mathrm{C} 6-\mathrm{O} 1$ & $1.433(4)$ & $\mathrm{N} 4-\mathrm{O} 2$ & $1.232(4)$ \\
$\mathrm{C} 6-\mathrm{C} 7$ & $1.502(4)$ & $\mathrm{C} 12-\mathrm{C} 7-\mathrm{C} 6$ & $1.237(3)$ \\
& & $\mathrm{C} 9-\mathrm{C} 8-\mathrm{C} 7$ & $118.9(2)$ \\
$\mathrm{C} 2-\mathrm{C} 1-\mathrm{N} 2$ & $108.0(3)$ & $\mathrm{C} 9-\mathrm{C} 8-\mathrm{H} 8$ & $120.3(3)$ \\
$\mathrm{C} 2-\mathrm{C} 1-\mathrm{N} 4$ & $126.9(3)$ & $\mathrm{C} 7-\mathrm{C} 8-\mathrm{H} 8$ & 119.8 \\
$\mathrm{~N} 2-\mathrm{C} 1-\mathrm{N} 4$ & $125.1(2)$ & $\mathrm{C} 10-\mathrm{C} 9-\mathrm{C} 8$ & 119.8 \\
$\mathrm{~N} 1-\mathrm{C} 2-\mathrm{C} 1$ & $109.1(3)$ & $\mathrm{C} 10-\mathrm{C} 9-\mathrm{H} 9$ & $119.4(3)$ \\
$\mathrm{N} 1-\mathrm{C} 2-\mathrm{H} 2$ & 125.4 & $\mathrm{C} 8-\mathrm{C} 9-\mathrm{H} 9$ & 120.3 \\
$\mathrm{C} 1-\mathrm{C} 2-\mathrm{H} 2$ & 125.4 & $\mathrm{C} 9-\mathrm{C} 10-\mathrm{C} 11$ & 120.3 \\
$\mathrm{~N} 1-\mathrm{C} 3-\mathrm{N} 2$ & $112.2(3)$ & $\mathrm{C} 11-\mathrm{C} 10-\mathrm{Br} 10$ & $121.8(3)$ \\
$\mathrm{N} 1-\mathrm{C} 3-\mathrm{C} 5$ & $119.7(2)$ & $\mathrm{C} 10-\mathrm{C} 11-\mathrm{C} 12$ & $119.4(2)$ \\
$\mathrm{N} 2-\mathrm{C} 3-\mathrm{C} 5$ & $128.1(3)$ & $118.8(2)$ \\
$\mathrm{N} 2-\mathrm{C} 4-\mathrm{H} 4 \mathrm{~A}$ & 109.5 & $118.1(3)$ \\
$\mathrm{N} 2-\mathrm{C} 4-\mathrm{H} 4 \mathrm{~B}$ & 109.5 & $\mathrm{H} 40.9$ \\
$\mathrm{H} 4 \mathrm{C} 4-\mathrm{H} 4 \mathrm{~B}$ & & &
\end{tabular}




\begin{tabular}{|c|c|c|c|}
\hline $\mathrm{N} 2-\mathrm{C} 4-\mathrm{H} 4 \mathrm{C}$ & 109.5 & $\mathrm{C} 12-\mathrm{C} 11-\mathrm{H} 11$ & 120.9 \\
\hline $\mathrm{H} 4 \mathrm{~A}-\mathrm{C} 4-\mathrm{H} 4 \mathrm{C}$ & 109.5 & $\mathrm{C} 11-\mathrm{C} 12-\mathrm{C} 7$ & $121.5(3)$ \\
\hline $\mathrm{H} 4 \mathrm{~B}-\mathrm{C} 4-\mathrm{H} 4 \mathrm{C}$ & 109.5 & $\mathrm{C} 11-\mathrm{C} 12-\mathrm{H} 12$ & 119.2 \\
\hline $\mathrm{N} 3-\mathrm{C} 5-\mathrm{C} 3$ & $123.6(3)$ & $\mathrm{C} 7-\mathrm{C} 12-\mathrm{H} 12$ & 119.2 \\
\hline $\mathrm{N} 3-\mathrm{C} 5-\mathrm{H} 5$ & 118.2 & $\mathrm{C} 3-\mathrm{N} 1-\mathrm{C} 2$ & $105.9(2)$ \\
\hline $\mathrm{C} 3-\mathrm{C} 5-\mathrm{H} 5$ & 118.2 & $\mathrm{C} 3-\mathrm{N} 2-\mathrm{C} 1$ & $104.7(2)$ \\
\hline $\mathrm{O} 1-\mathrm{C} 6-\mathrm{C} 7$ & $108.3(2)$ & $\mathrm{C} 3-\mathrm{N} 2-\mathrm{C} 4$ & $126.7(3)$ \\
\hline $\mathrm{O} 1-\mathrm{C} 6-\mathrm{H} 6 \mathrm{~A}$ & 110.0 & $\mathrm{C} 1-\mathrm{N} 2-\mathrm{C} 4$ & $128.6(2)$ \\
\hline $\mathrm{C} 7-\mathrm{C} 6-\mathrm{H} 6 \mathrm{~A}$ & 110.0 & $\mathrm{C} 5-\mathrm{N} 3-\mathrm{O} 1$ & $110.1(2)$ \\
\hline $\mathrm{O} 1-\mathrm{C} 6-\mathrm{H} 6 \mathrm{~B}$ & 110.0 & $\mathrm{O} 3-\mathrm{N} 4-\mathrm{O} 2$ & $123.5(3)$ \\
\hline $\mathrm{C} 7-\mathrm{C} 6-\mathrm{H} 6 \mathrm{~B}$ & 110.0 & $\mathrm{O} 3-\mathrm{N} 4-\mathrm{C} 1$ & $117.4(2)$ \\
\hline $\mathrm{H} 6 \mathrm{~A}-\mathrm{C} 6-\mathrm{H} 6 \mathrm{~B}$ & 108.4 & $\mathrm{O} 2-\mathrm{N} 4-\mathrm{C} 1$ & $119.1(3)$ \\
\hline $\mathrm{C} 8-\mathrm{C} 7-\mathrm{C} 12$ & $118.9(3)$ & $\mathrm{N} 3-\mathrm{O} 1-\mathrm{C} 6$ & $108.3(2)$ \\
\hline $\mathrm{C} 8-\mathrm{C} 7-\mathrm{C} 6$ & $122.2(3)$ & & \\
\hline $\mathrm{N} 2-\mathrm{C} 1-\mathrm{C} 2-\mathrm{N} 1$ & $0.2(3)$ & $\mathrm{C} 5-\mathrm{C} 3-\mathrm{N} 1-\mathrm{C} 2$ & $178.5(3)$ \\
\hline $\mathrm{N} 4-\mathrm{C} 1-\mathrm{C} 2-\mathrm{N} 1$ & $-179.8(3)$ & $\mathrm{C} 1-\mathrm{C} 2-\mathrm{N} 1-\mathrm{C} 3$ & $0.5(3)$ \\
\hline $\mathrm{N} 1-\mathrm{C} 3-\mathrm{C} 5-\mathrm{N} 3$ & $-170.9(3)$ & $\mathrm{N} 1-\mathrm{C} 3-\mathrm{N} 2-\mathrm{C} 1$ & $1.3(3)$ \\
\hline $\mathrm{N} 2-\mathrm{C} 3-\mathrm{C} 5-\mathrm{N} 3$ & $8.7(5)$ & $\mathrm{C} 5-\mathrm{C} 3-\mathrm{N} 2-\mathrm{C} 1$ & $-178.3(3)$ \\
\hline $\mathrm{O} 1-\mathrm{C} 6-\mathrm{C} 7-\mathrm{C} 8$ & $-23.1(4)$ & $\mathrm{N} 1-\mathrm{C} 3-\mathrm{N} 2-\mathrm{C} 4$ & $-177.1(3)$ \\
\hline $\mathrm{O} 1-\mathrm{C} 6-\mathrm{C} 7-\mathrm{C} 12$ & $159.4(3)$ & $\mathrm{C} 5-\mathrm{C} 3-\mathrm{N} 2-\mathrm{C} 4$ & $3.3(5)$ \\
\hline $\mathrm{C} 12-\mathrm{C} 7-\mathrm{C} 8-\mathrm{C} 9$ & $-0.1(4)$ & $\mathrm{C} 2-\mathrm{C} 1-\mathrm{N} 2-\mathrm{C} 3$ & $-0.9(3)$ \\
\hline $\mathrm{C} 6-\mathrm{C} 7-\mathrm{C} 8-\mathrm{C} 9$ & $-177.7(3)$ & $\mathrm{N} 4-\mathrm{C} 1-\mathrm{N} 2-\mathrm{C} 3$ & $179.2(3)$ \\
\hline $\mathrm{C} 7-\mathrm{C} 8-\mathrm{C} 9-\mathrm{C} 10$ & $0.0(5)$ & $\mathrm{C} 2-\mathrm{C} 1-\mathrm{N} 2-\mathrm{C} 4$ & $177.4(3)$ \\
\hline $\mathrm{C} 8-\mathrm{C} 9-\mathrm{C} 10-\mathrm{C} 11$ & $0.5(5)$ & $\mathrm{N} 4-\mathrm{C} 1-\mathrm{N} 2-\mathrm{C} 4$ & $-2.5(5)$ \\
\hline $\mathrm{C} 8-\mathrm{C} 9-\mathrm{C} 10-\mathrm{Br} 1$ & $-178.8(2)$ & $\mathrm{C} 3-\mathrm{C} 5-\mathrm{N} 3-\mathrm{O} 1$ & $179.2(2)$ \\
\hline $\mathrm{C} 9-\mathrm{C} 10-\mathrm{C} 11-\mathrm{C} 12$ & $-0.9(4)$ & $\mathrm{C} 2-\mathrm{C} 1-\mathrm{N} 4-\mathrm{O} 3$ & $6.0(5)$ \\
\hline $\mathrm{Br} 1-\mathrm{C} 10-\mathrm{C} 11-\mathrm{C} 12$ & $178.4(2)$ & $\mathrm{N} 2-\mathrm{C} 1-\mathrm{N} 4-\mathrm{O} 3$ & $-174.0(3)$ \\
\hline $\mathrm{C} 10-\mathrm{C} 11-\mathrm{C} 12-\mathrm{C} 7$ & $0.8(4)$ & $\mathrm{C} 2-\mathrm{C} 1-\mathrm{N} 4-\mathrm{O} 2$ & $-174.7(3)$ \\
\hline $\mathrm{C} 8-\mathrm{C} 7-\mathrm{C} 12-\mathrm{C} 11$ & $-0.3(4)$ & $\mathrm{N} 2-\mathrm{C} 1-\mathrm{N} 4-\mathrm{O} 2$ & $5.3(4)$ \\
\hline $\mathrm{C} 6-\mathrm{C} 7-\mathrm{C} 12-\mathrm{C} 11$ & $177.3(3)$ & $\mathrm{C} 5-\mathrm{N} 3-\mathrm{O} 1-\mathrm{C} 6$ & $-171.8(2)$ \\
\hline $\mathrm{N} 2-\mathrm{C} 3-\mathrm{N} 1-\mathrm{C} 2$ & $-1.2(3)$ & $\mathrm{C} 7-\mathrm{C} 6-\mathrm{O} 1-\mathrm{N} 3$ & $179.7(2)$ \\
\hline
\end{tabular}

Hydrogen-bond geometry $\left(\AA,{ }^{\circ}\right)$

\begin{tabular}{lllll}
\hline$D-\mathrm{H} \cdots A$ & $D-\mathrm{H}$ & $\mathrm{H} \cdots A$ & $D \cdots A$ & $D-\mathrm{H} \cdots A$ \\
\hline $\mathrm{C} 4-\mathrm{H} 4 C \cdots \mathrm{N} 3$ & 0.98 & 2.24 & $2.997(4)$ & 133 \\
$\mathrm{C} 4-\mathrm{H} 4 A \cdots \mathrm{N} 1^{\mathrm{i}}$ & 0.98 & 2.62 & $3.499(4)$ & 149 \\
$\mathrm{C} 9-\mathrm{H} 9 \cdots \mathrm{N} 1^{\mathrm{ii}}$ & 0.95 & 2.77 & $3.681(4)$ & 160 \\
$\mathrm{C} 2-\mathrm{H} 2 \cdots 3^{\mathrm{iii}}$ & 0.95 & 2.44 & $3.282(4)$ & 148 \\
$\mathrm{C} 5-\mathrm{H} 5 \cdots \mathrm{O} 2^{\mathrm{iv}}$ & 0.95 & 2.64 & $3.341(4)$ & 131 \\
$\mathrm{C} 6-\mathrm{H} 6 A \cdots \mathrm{O} 2^{\mathrm{v}}$ & 0.99 & 2.63 & $3.254(4)$ & 121 \\
$\mathrm{C} 4-\mathrm{H} 4 C \cdots \mathrm{Br}^{\mathrm{vi}}$ & 0.98 & 2.85 & $3.491(3)$ & 124
\end{tabular}

Symmetry codes: (i) $-x+1 / 2, y+1 / 2,-z+1 / 2$; (ii) $x-1 / 2,-y+1 / 2, z+1 / 2$; (iii) $-x+1,-y+1,-z$; (iv) $-x+1 / 2, y-1 / 2,-z+1 / 2$; (v) $x-1 / 2,-y+3 / 2, z+1 / 2$; (vi) $-x+1 / 2, y+1 / 2,-z+3 / 2$. 